\title{
Star formation in the outer Galaxy
}

\author{
J.G.A. Wouterloot ${ }^{1}$, J. Brand ${ }^{1,2,3}$, and C. Henkel ${ }^{1}$ \\ ${ }^{1}$ Max-Planck-Institut für Radioastronomie, Auf dem Hügel 69, D-5300 Bonn 1, Federal Republic of Germany \\ ${ }^{2}$ Sterrewacht, Postbus 9513, NL-2300 RA Leiden, The Netherlands \\ ${ }^{3}$ Astronomy Program, University of Maryland, College Park, MD 20742, USA
}

Received March 10, accepted August 12, 1987

Summary. We have used the $100 \mathrm{~m}$ Effelsberg telescope to search for $\mathrm{H}_{2} \mathrm{O}$ and $\mathrm{OH}$ maser emission toward IRAS point sources in the neighbourhood of $\mathrm{H}$ il regions with galactocentric distances larger than $14 \mathrm{kpc}\left(R_{0}=10 \mathrm{kpc}\right)$. We found $15 \mathrm{H}_{2} \mathrm{O}$ masers and $1 \mathrm{OH}$ maser in the outer Galaxy and in addition $10 \mathrm{H}_{2} \mathrm{O}$ masers and $1 \mathrm{OH}$ maser in some other regions closer to the solar circle. Both the FIR luminosity distribution of sources in the outer Galaxy and the average number of FIR sources per $H$ il region with $L_{\mathrm{IR}}>10^{4} L_{\odot}$ are the same as in the Perseus arm. This suggests that once star formation started, the process continues similarly as in a cloud located in a spiral arm. The amount of star formation per unit mass of $\mathrm{H}_{2}$ at $R=15 \mathrm{kpc}$ is equal to that in the solar neighbourhood. Eight of the $\mathrm{H}$ II regions were observed with the VLA at $6 \mathrm{~cm}$. The fields contain 18 radio sources, 9 of which are associated with an IRAS source. Five IRAS sources in these fields show no radio emission. High angular resolution $\mathrm{CO}$ data from the maser positions in the outer Galaxy show a good agreement between $\mathrm{CO}$ and $\mathrm{H}_{2} \mathrm{O}$ velocities. Toward the masers in $\mathrm{S} 270$ and S 209, weak emission in the linewings is detected over a velocity range of $20-30 \mathrm{~km} \mathrm{~s}^{-1}$ providing, for the first time, evidence for molecular outflows at large $(R=20 \mathrm{kpc})$ galactic radii.

Key words: $\mathrm{H}_{2} \mathrm{O}$ masers - infrared sources - star formation - $\mathrm{H}$ II regions - radio continuum sources

\section{Introduction}

Massive stars $\left(M>6.5 M_{\odot}\right.$; spectral type earlier than B 3) are formed at distances from the galactic centre $(R)$ up to about $20 \mathrm{kpc}$, as can be concluded from the presence of $\mathrm{H}$ iI regions (see Fich and Blitz, 1984; Brand, 1986). However, the properties of objects (clouds, $\mathrm{H}$ il regions) and the relative importance of the various processes that induce cloud and star formation in the outer Galaxy (here defined as $R>14 \mathrm{kpc}$ ) may be systematically different from those in the inner Galaxy.

To study star formation in the outer Galaxy and to get insight into these differences, we looked for $\mathrm{H}_{2} \mathrm{O}$ and $\mathrm{OH}$ masers in and around $\mathrm{H}$ il regions at large galactocentric distances. $\mathrm{No}_{2} \mathrm{O}$ masers are known beyond one near S 128 (Ho et al., 1981) at $R=14.1 \pm 2.0 \mathrm{kpc}$ (Fich and Blitz, 1984). Searches for other

Send offprint requests to: J.G.A. Wouterloot maser sources with relatively low sensitivity in distant $\mathrm{CO}$ clouds were negative (Henkel et al., 1986). Wouterloot and Walmsley (1986) showed that in general all $\mathrm{H}_{2} \mathrm{O}$ masers in regions of star formation are associated with a FIR point source with specific colours in the IRAS catalogue. This result was used for the selection of our objects in the present study. As is known from numerous investigations (e.g. Blitz, 1980), most $\mathrm{H}$ il regions are associated with molecular clouds which have sizes of typically 10 $50 \mathrm{pc}$. We have studied star formation in the clouds associated with $\mathrm{H}$ II regions in the outer Galaxy by selecting IRAS sources in a one square degree field around these $\mathrm{H}$ II regions. We compare and discuss the presence (relative number density) of detected maser sources and the flux density distribution of IR sources for various parts of the Galaxy.

For a number of sources we also obtained $6 \mathrm{~cm}$ radio continuum data to investigate the size, morphology and flux density of radio emission associated with the $\mathrm{H}$ II regions and the FIR sources and to compare these data with those from sources in the inner part of the Galaxy.

We assumed a distance to the galactic centre $\left(R_{0}\right)$ of $10 \mathrm{kpc}$. Our conclusions will not change if a value of $8.5 \mathrm{kpc}$ is used.

\section{Source selection and observations}

We selected regions to be studied primarily from the catalogue of Blitz, Fich and Stark (1982; hereafter called BFS). The criterion was $R$ that should be larger than $14 \mathrm{kpc}$. Additionally we selected from the same catalogue more nearby, small $\mathrm{H}$ iI regions with a high $\mathrm{CO}$ line temperature. We also observed three regions from Brand, Blitz and Wouterloot (1986; denoted as BBW), with a large (kinematic) distance. Around each of the regions, we selected positions to be observed in $\mathrm{H}_{2} \mathrm{O}$ from the IRAS point source catalogue in a one square degree field centered on the $\mathrm{H}$ II region. For the sources with a large heliocentric distance $(d>2-3 \mathrm{kpc})$, this field should include the whole cloud associated with the $\mathrm{H}$ II regions. Selected sources had less than two upper limits in the four fluxes, and colours in the range where maser sources are found (see Wouterloot and Walmsley, 1986, Fig. 3): $-0.1<R_{12}<1.5$, $-0.25<R_{23}<1.5$ and $-0.5<R_{34}<0.261+0.227 R_{23}$, where $R_{\mathrm{ij}}$ is the ratio of $\log (v S)$ in band $i$ and band $j$.

The positions with detected maser sources were then observed in the $18 \mathrm{~cm} \mathrm{OH}$ main lines and some $\mathrm{H}$ in regions [most of them at very large $R(>17 \mathrm{kpc})$ ], were also observed at $6 \mathrm{~cm}$ wavelength 
Table 1. Areas observed at $6 \mathrm{~cm}$ with the VLA

\begin{tabular}{|c|c|c|c|}
\hline \multirow{2}{*}{$\begin{array}{l}\text { Field } \\
\text { (1) }\end{array}$} & \multicolumn{3}{|c|}{ Coordinates of map centers } \\
\hline & $\begin{array}{l}\text { RA } \quad(1950.0) \\
(2)\end{array}$ & $\begin{array}{l}\text { Decl. } \\
\text { (3) }\end{array}$ & $\begin{array}{l}\text { rms } \\
(\mathrm{mJy}) \\
(4)\end{array}$ \\
\hline S 259 & 60835.0 & 172700 & 0.5 \\
\hline \multirow[t]{3}{*}{ S 266} & 61433.0 & 145600 & 0.3 \\
\hline & 61550.0 & 150600 & 0.7 \\
\hline & 61550.0 & 151800 & 0.7 \\
\hline \multirow[t]{2}{*}{ S 270} & 60724.0 & 124900 & 0.3 \\
\hline & 60801.0 & 123300 & 0.2 \\
\hline S 283 & 63554.0 & 004600 & 0.3 \\
\hline S 286 & 65206.0 & -42800 & 0.2 \\
\hline BBW 33 & 72755.0 & -203800 & 0.4 \\
\hline BBW 44/45 & 73108.0 & -220300 & 0.3 \\
\hline BBW 66 & 74257.0 & -252400 & 0.8 \\
\hline
\end{tabular}

with the Very Large Array (VLA) of the National Radio Astronomy Observatory ${ }^{1}$.

The $\mathrm{H}_{2} \mathrm{O}$ maser observations were made between April 3, 1985 and November 18, 1986 with the $100 \mathrm{~m}$ radio telescope in Effelsberg (HPBW 40"). All positions with detected $\mathrm{H}_{2} \mathrm{O}$ masers were observed at least twice. We used a liquid-He cooled maser receiver which had a system temperature at the zenith of about 50-90 K depending on whether conditions. We observed with a 1024 channel autocorrelator split into two equal parts with bandwidths of 6.25 and $25 \mathrm{MHz}$. At $22.235 \mathrm{GHz}$ this provides channel spacings of $0.16 \mathrm{~km} \mathrm{~s}^{-1}$ and $0.66 \mathrm{~km} \mathrm{~s}^{-1}$ and velocity coverages of $82 \mathrm{~km} \mathrm{~s}^{-1}$ and $330 \mathrm{~km} \mathrm{~s}^{-1}$ respectively. The typical integration time was 3 minutes, which resulted in an rms noise level in the high resolution spectra between $0.15 \mathrm{Jy}$ and $0.50 \mathrm{Jy}$, depending on weather and elevation. At positions where a signal was detected, we made a 5-point cross to derive a position with an accuracy of about $5^{\prime \prime}$. Calibration sources used are NGC 7027, 3 C 123 and 3 C 286, with flux densities adopted from Baars et al. (1977). The accuracy of the derived $\mathrm{H}_{2} \mathrm{O}$ flux densities is $10-20 \%$.

On January 17, 18, 25, and 26, 1986 we observed the detected $\mathrm{H}_{2} \mathrm{O}$ masers in $\mathrm{OH}$ with the $100 \mathrm{~m}$ telescope to search for $1665 \mathrm{MHz}$ maser emission. We used a paramp receiver with a system temperature of about $60 \mathrm{~K}$. The beam at $18 \mathrm{~cm}$ is 7.8 . LC and RC polarization were observed simultaneously with a velocity resolution of $0.5 \mathrm{~km} \mathrm{~s}^{-1}$. The rms sensitivity was about $0.03 \mathrm{Jy}$ (for unpolarized radiation). For detected $\mathrm{OH}$ masers, a position was derived with an accuracy of about $30^{\prime \prime}$.

Most of the maser positions in the outer Galaxy were also observed in the $J=1-0$ line of $\mathrm{CO}(115.271 \mathrm{GHz})$ with the $30 \mathrm{~m}$ IRAM telescope. The data were collected with an SIS receiver $(150 \mathrm{~K}$ receiver noise) in the position switching mode, with a spectrometer consisting of $2561 \mathrm{MHz}$ and $256100 \mathrm{KHz}$ channels. The beam size was 21". Calibration was obtained toward IRc 2 in Orion, for which we assumed $T_{\mathrm{A}}^{*}=70 \mathrm{~K}$ (for details, see Wilson et al., 1986).

Continuum observations at $6 \mathrm{~cm}$ wavelength of the regions listed in Table 1 were made on February 5, 1986 with the VLA in

${ }^{1}$ The National Radio Astronomy Observatory is operated by Associated Universities, Inc., under contract with the National Science Foundation the $\mathrm{D}$ configuration at the beginning of the transition to A array. We used 24 antennas, a bandwidth of $100 \mathrm{MHz}$ and observed each region for $2 \times 10$ minutes. The rms noise in our maps (column 4 in Table 1) is 0.3 to $0.8 \mathrm{mJy}$. During part of the observations, one half of the correlators was inoperable and the bandwidth was reduced to $50 \mathrm{MHz}$. The synthesized beam, using uniform weighting, is 17 ". $6 \times 15$ ".5. The flux density was calibrated using the source $3 \mathrm{C} 48$ and adopting a flux density of $5.575 \mathrm{Jy}$. Two of the H II regions showed maser emission at more than $10^{\prime}$ from the optical position. In these cases, we observed more than one field. In total, 11 fields were observed. The data were reduced in the standard way using the AIPS programs installed in Leiden.

\section{Observational results}

\subsection{Effelsberg $\mathrm{H}_{2} \mathrm{O}$ observations}

The IRAS sources observed in $\mathrm{H}_{2} \mathrm{O}$ are listed in Table 2. In column 1 we give the name of the $\mathrm{H}_{\text {II }}$ region. In column 2 the galactocentric distance $(R)$ and in column 3 the heliocentric distance $(d)$. In column 4 is listed the name of the IRAS source, in column 5 the rms noise in the high resolution data and in column 6 the dates of observation if no line was detected.

In total we observed 139 sources near 45 nebulae; 77 of these sources, in the neighbourhood of 29 nebulae, have $R>14 \mathrm{kpc}$. No known FIR sources are located within $30^{\prime}$ from S 271 . We detected 25 masers: 15 toward sources with $R>14 \mathrm{kpc}$ and 10 toward sources with smaller galactocentric distances. The distribution of the $\mathrm{H}_{2} \mathrm{O}$ maser sources projected on the galactic plane is displayed in Fig. 1.

The detection rates for the regions with $R>14 \mathrm{kpc}$ and $R<14 \mathrm{kpc}$ are very similar: $19 \%$ versus $16 \%$. Some of the sources were observed several times. We repeated the observations of all the 25 detected masers (first detections were obtained between April and December 1985) on November 18 1986. Eight of them were not seen, indicating strong variability on a timescale of months. The spectra are shown in Fig. 2.

There is one IRAS source where we found even two $\mathrm{H}_{2} \mathrm{O}$ sources: IRAS $21418+6552$ in BFS 11 . The masers toward S 128 and S 269 were already known before (see Ho et al., 1981; Wouterloot and Walmsley, 1986).

Some of the FIR sources, where $\mathrm{H}_{2} \mathrm{O}$ was not detected, could be background galaxies. The number of sources with the selected colours at high galactic latitudes is about one per 25 square degrees; hence we would expect that about 2 of the 139 sources are background galaxies. Whether an individual source is associated with one of the $\mathrm{H}$ II regions can only be decided, when e.g. $\mathrm{CO}$ velocities are obtained. Observational parameters of the detected sources are shown in Table 3. Each row gives information on data obtained on a different date. Column 1 gives the name of the $\mathrm{H}$ II region. Columns 2 to 5 give the position of the IRAS source and the derived $\mathrm{H}_{2} \mathrm{O}$ position. In columns 6 and 7 we list the peak and the integrated $\mathrm{H}_{2} \mathrm{O}$ flux density. Columns 8 to 11 show the velocity of the $\mathrm{H}_{2} \mathrm{O}$ peak, the range of $\mathrm{H}_{2} \mathrm{O}$ emission ( $V_{\text {low }}$ to $V_{\text {high }}$ ) and the velocity of the molecular cloud near the maser source or the $\mathrm{H}$ II region (see Sect. 3.3; otherwise see BFS and Brand et al., 1987). The latter two references provide not necessarily the velocity at the position of the maser source). Columns 12 and 13 give the integrated $\mathrm{H}_{2} \mathrm{O}$ luminosity $\left(1.810^{-7}\right.$ noted $\left.1.8-7\right)$ and the FIR luminosity $\left(1.310^{4}\right.$ noted 1.34 ; derived, assuming an emissivity proportional to frequency, correcting the IRAS fluxes for derived colour temperatures between two adjacent bands and extrapolat- 


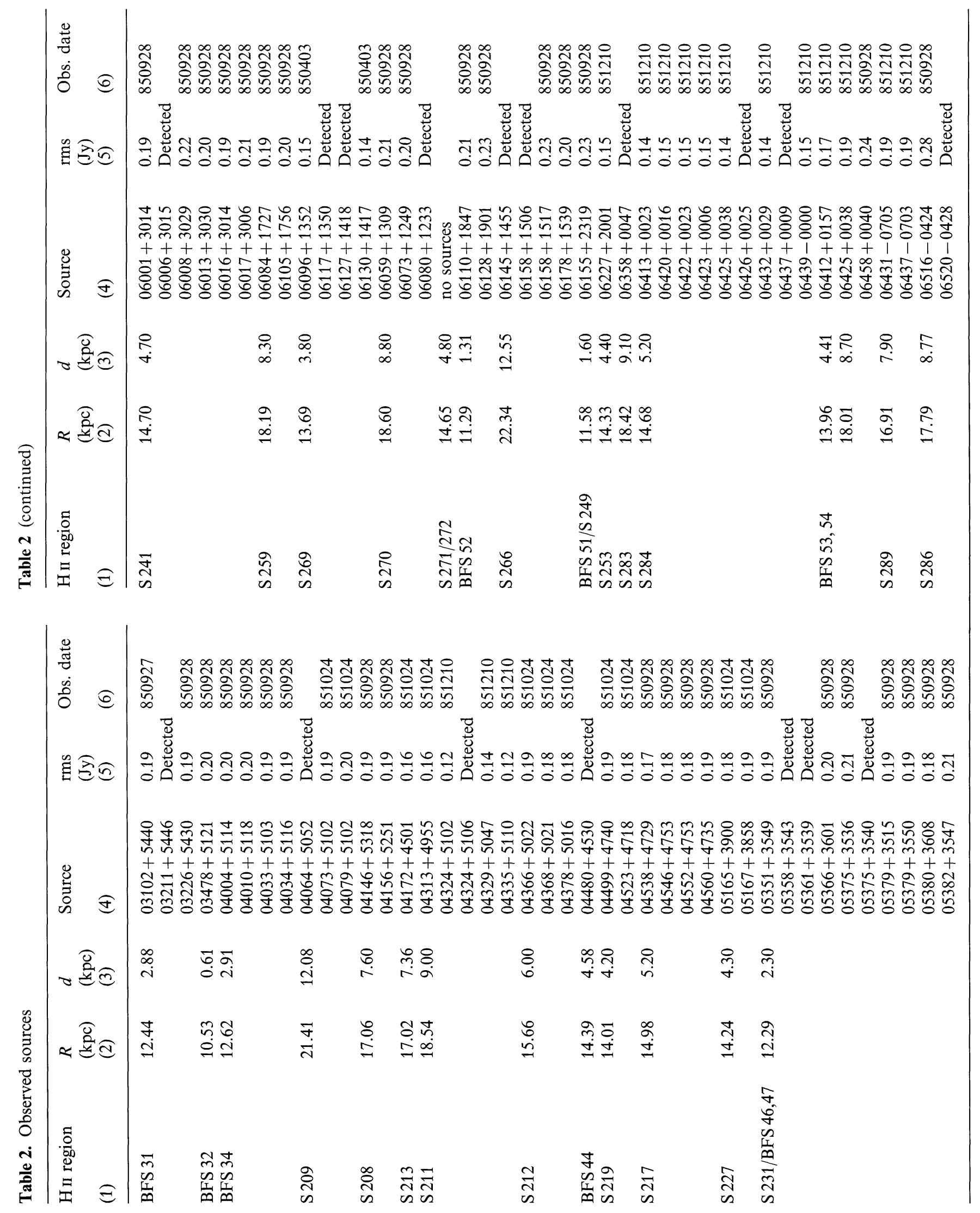




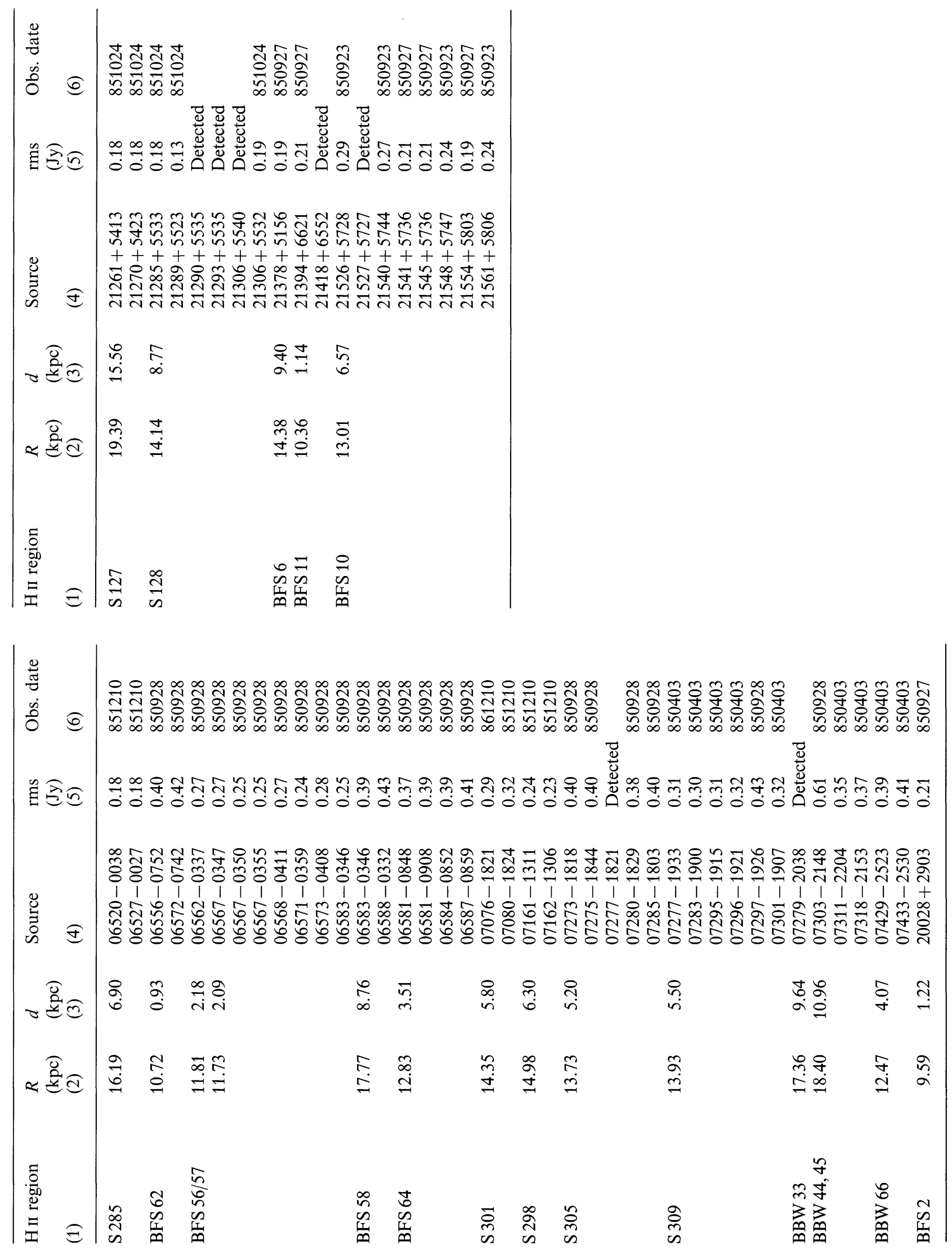




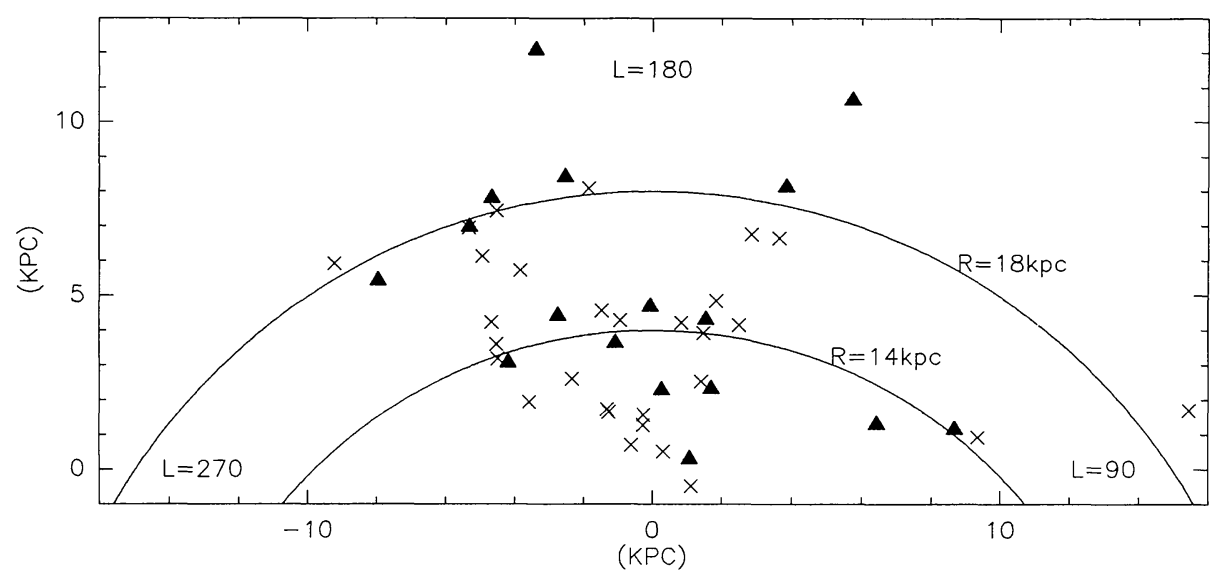

Fig. 1. The location of the $\mathrm{H}_{2} \mathrm{O}$ maser sources (filled triangles), projected on the galactic plane. Crosses indicate $\mathrm{H}$ II regions, where no $\mathrm{H}_{2} \mathrm{O}$ emission has been found. The Sun is at $(0,0)$ ing the fluxes to 6 and $400 \mu \mathrm{m}$ ). Column 14 gives the date of the observation.

\subsection{Effelsberg $\mathrm{OH}$ observations}

Towards the $\mathrm{H}_{2} \mathrm{O}$ maser sources, we detected two new $1665 \mathrm{MHz}$ OH masers, a detection rate of only $8 \%$. One of them (near $\mathrm{S} 128$ at $R=14.1 \mathrm{kpc}$ ) is located in the outer Galaxy. The spectra are shown in Fig. 3. Especially for weak $\mathrm{H}_{2} \mathrm{O}$ masers, the $\mathrm{OH}$ maser detection rate is very low. The ratio of $\mathrm{H}_{2} \mathrm{O} / \mathrm{OH}$ maser strength can in some cases be 100 (Caswell et al., 1983) and hence our low success rate in $\mathrm{OH}$ is not surprising. All components in both $\mathrm{OH}$ masers are $100 \%$ circularly polarized (see Fig. 3), which is normal for type I masers. We also observed these sources at $1667 \mathrm{MHz}$ but no line was detected.

\subsection{IRAM CO observations}

Toward 10 of the 15 masers in the outer Galaxy, high angular resolution $\mathrm{CO}(J=1-0)$ spectra were taken which are shown in Fig. 4. The mean difference between $\mathrm{H}_{2} \mathrm{O}$ and $\mathrm{CO}$ peak velocities in this sample is $0.9 \pm 0.9 \mathrm{~km} \mathrm{~s}^{-1}$. Toward at least two of the sources, S 209 and S 270, weak line wings are observed over a velocity range of 20 and $30 \mathrm{~km} \mathrm{~s}^{-1}$ respectively, suggesting the presence of molecular outflows. Hence these two sources may represent the first examples of this phenomenon at galactocentric distances of about $20 \mathrm{kpc}$. A further possible candidate is $S 241$ at $R=15 \mathrm{kpc}$, but here one of the line wings is blended with emission from a second velocity component.

\subsection{VLA $6 \mathrm{~cm}$ continuum observations}

Within the 11 VLA fields, covering the 8 regions listed in Table 1 , radio emission was detected at 19 positions. Observed parameters of sources detected with the VLA and $(1 \sigma)$ upper limits at positions of non-detected IRAS sources are listed in Table 4. In column 1 we give a sequential number to identify the IRAS/radio sources near each $\mathrm{H}$ il region. Columns 2 and 3 give the position of the FIR source, columns 4 and 5 list the radio position. Columns 6 to 8 summarize the radio results and give the peak flux density (column 6), the integrated flux density (column 7) with their respective uncertainties, and the angular size of the radio source (column 8). When the emission was not resolved with the VLA, it is marked "nr" in this column. Eight radio sources, five of which are resolved, coincide with an optical nebulosity. Only BBW 66 has no radio counterpart; it may be a reflection nebula rather than an $\mathrm{H}$ II region. One radio source (S 266-3) coincides with a FIR source, but not with an optical nebula and may be an optically highly obscured object. There are ten radio sources that do not coincide with either an optical nebula, FIR- or $\mathrm{H}_{2} \mathrm{O}$ maser source; these are most likely background sources unrelated to the $\mathrm{H}$ II regions [the expected number of random background sources in the 11 VLA fields within 8.5 of the field centres down to a level of $1.5 \mathrm{mJy}$ is $12 \pm 3.5$ (making use of data by Maslowski et al., 1984 and Wall et al., 1982)]. A total of 14 FIR sources are located within our VLA fields, nine of which coincide with a radio source. The FIR sources which are not detected at $6 \mathrm{~cm}$ generally have a luminosity of less than $610^{3} L_{\odot}$. The exception is S 266-1, which has a luminosity of $2.110^{4} L_{\odot}$.

Maps of the most interesting regions are shown in Fig. 5a-f. In these figures we also marked the positions of the FIR sources and $\mathrm{H}_{2} \mathrm{O}$ masers (if present). These maps are not corrected for primary beam attenuation. Detailed comments are given in Sect. 4.

Parameters, derived from radio and FIR data, are collected in Table 5. In this table, column 1 identifies, for each $\mathrm{H}$ in region, the FIR/radio sources (see Table 4). Column 2 lists the excitation parameter $u\left(u\left(\mathrm{pccm}^{-2}\right)=13.5(d(\mathrm{kpc}))^{2 / 3}(S(\mathrm{Jy}))^{1 / 3}\right)$ and its rms uncertainty, where $d$ is the heliocentric distance and $S$ the integrated flux density. Column 3 gives the linear size of each $\mathrm{H}_{\text {II }}$ region and column 4 the electron density $n_{\mathrm{e}}$ in the ionized region $\left(n_{\mathrm{e}}\left(\mathrm{cm}^{-3}\right)=2569.6(S(\mathrm{Jy}))^{1 / 2}(d(\mathrm{kpc}))^{-1 / 2}(\theta(\operatorname{arcmin}))^{-1 / 2}\right.$, where $\theta$ is the angular diameter of the H II region; Fich, 1983). In column 5 the IR luminosity of the IRAS source is given. In column 6 we present the "best fit" spectral type that corresponds to the excitation parameter and $L_{\mathrm{FIR}}$ (see below); $V_{0}$ indicates ZAMS luminosity class. Column 7 lists the heliocentric distance (with its uncertainty). Column 8 indicates whether the distance is photometric ("p") or kinematic ("k"); using $R_{0}=10 \mathrm{kpc}$, $\theta_{0}=250 \mathrm{~km} \mathrm{~s}^{-1}$ and the rotation curve by Fich and Blitz (1984). For all kinematic distances an uncertainty of $25 \%$ was adopted. The distance to S 259 is based on a single star (taken from Moffat et al., 1979) and a $25 \%$ uncertainty was adopted here as well.

The excitation parameter depends on the number of Lycontinuum photons emitted by the exciting star(s) of the $\mathrm{H}$ II region. Assuming all these photons are used to ionize the gas surrounding the star(s), $u$ is an indicator of their (average) spectral type. Similarly the FIR luminosity can be used to determine the total stellar luminosity, when it is assumed that all photons emitted by the star(s) heat up the surrounding gas and dust and eventually are reemitted in the infrared. To relate $u$ and $L_{\mathrm{IR}}$ to 

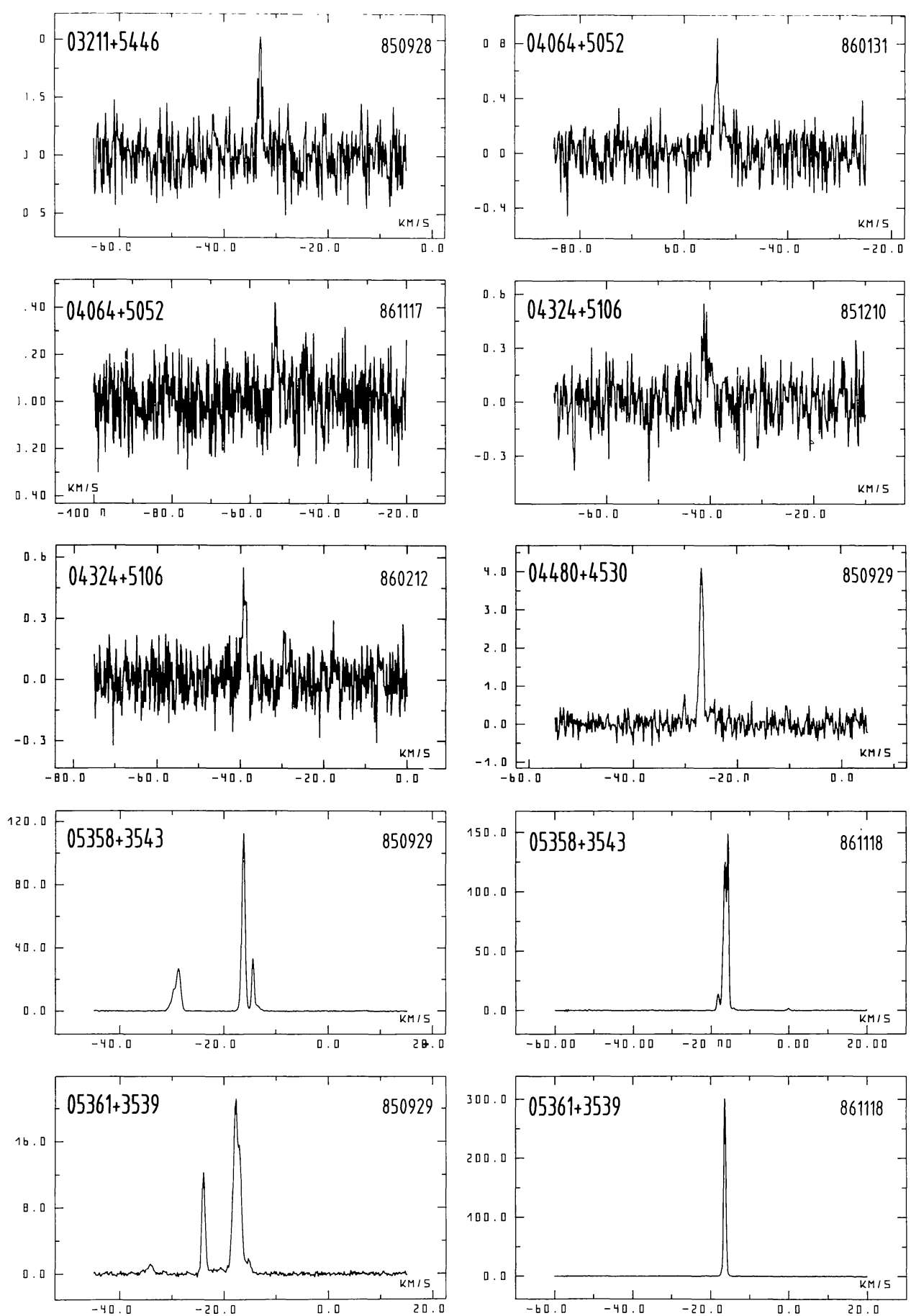

Fig. 2. Spectra of $\mathrm{H}_{2} \mathrm{O}$ masers. The scale is in Jy and velocities are with respect to the local standard of rest. The spectrum of $\mathrm{S} 269(06117+1350)$ is published in Wouterloot and Walmsley (1986)

spectral type we have made use of the tables by Panagia (1973). Combining the radio and IR data we arrived at the "best fit" spectral type, given in Table 5 . This procedure assumes excitation by a single star. In cases where there is only either IR or radio emission, the spectral type was derived assuming the star to be on the main sequence. All radio sources are excited by the ionizing equivalent of early B stars (only S 259 would house an O 9.5 star). As noted above, radio sources not associated with either an optical nebula, FIR- or $\mathrm{H}_{2} \mathrm{O}$ maser source are probably background objects and the derivation of spectral types from $u$ is a purely academic excercise in these cases. Optical data are available for a few $\mathrm{H}$ II regions. Comparing the spectral types determined at optical and radio wavelengths, one notes that the $u$ (optical data) $<u$ (radio data), suggesting that there are unseen sources of ionization in the $\mathrm{H}$ II regions under consideration. 

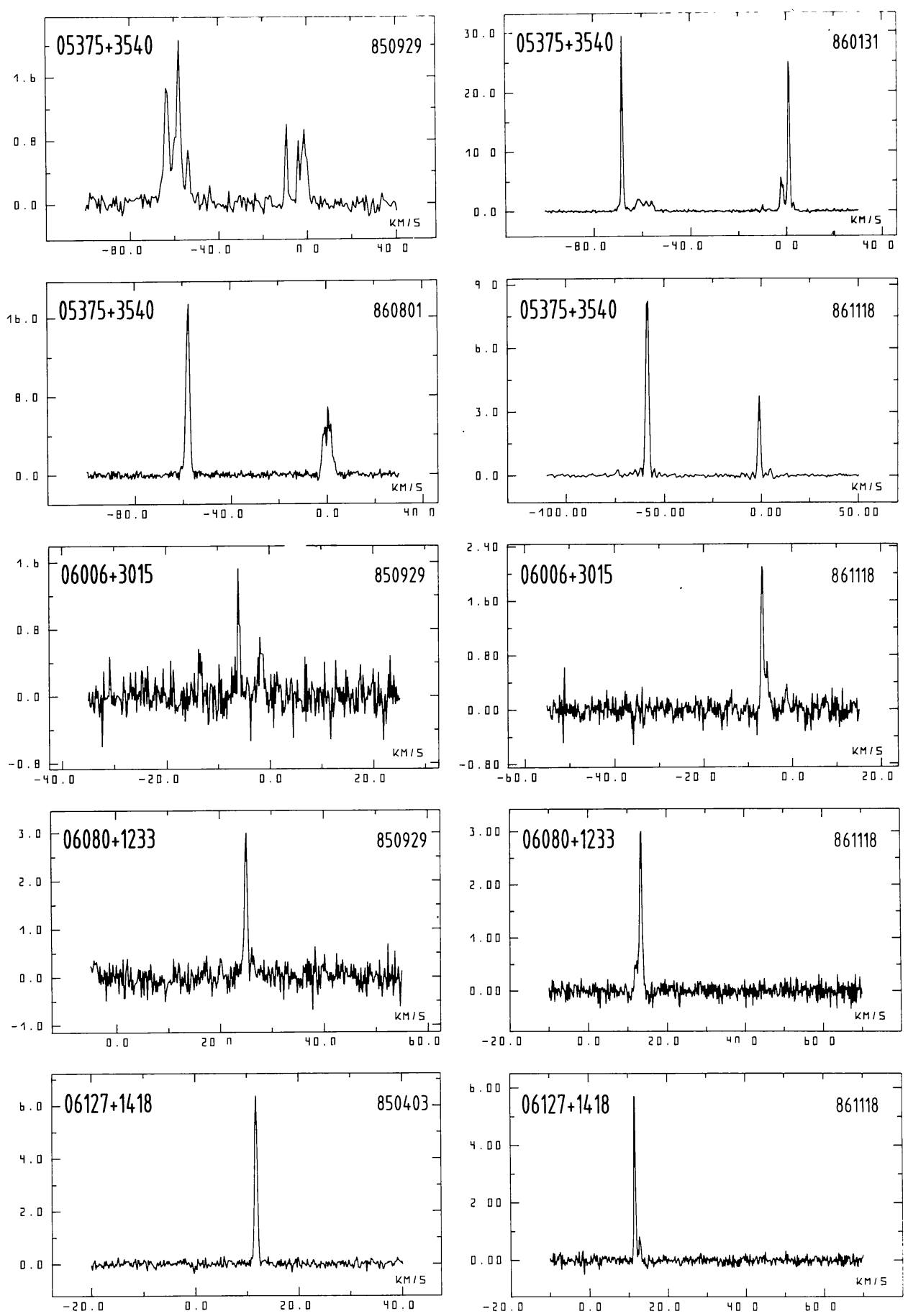

Fig. 2 (continued)

\section{Notes on individual sources}

In most cases the masers are within some tens of parsecs of the optical position of the $\mathrm{HI}$ region. For the sources near BFS 44, $\mathrm{S} 286$, and BBW 33 these projected distances are even less than 1 pc. On the other hand the source $06145+1155$ near S 266 is at $117 \mathrm{pc}$ from the $\mathrm{H}$ II region if we assume a kinematic distance of $12.55 \mathrm{kpc}$. The velocity of the maser is close to the $\mathrm{CO}$ velocity.
This suggests that either the distance is overestimated by at least a factor of two or that two separate complexes exist such as in Orion (Orion A and B). For some regions, e.g. in the case of BFS 53/54 and $S 211 / 212$, which are less than one degree apart, the assignment to one or the other $\mathrm{H}$ II region can be difficult.

BFS 31: The maser detected on 850928 near $03211+5446$ was not seen on 851024 . However the velocity of the line was $-33 \mathrm{~km} \mathrm{~s}^{-1}$, 

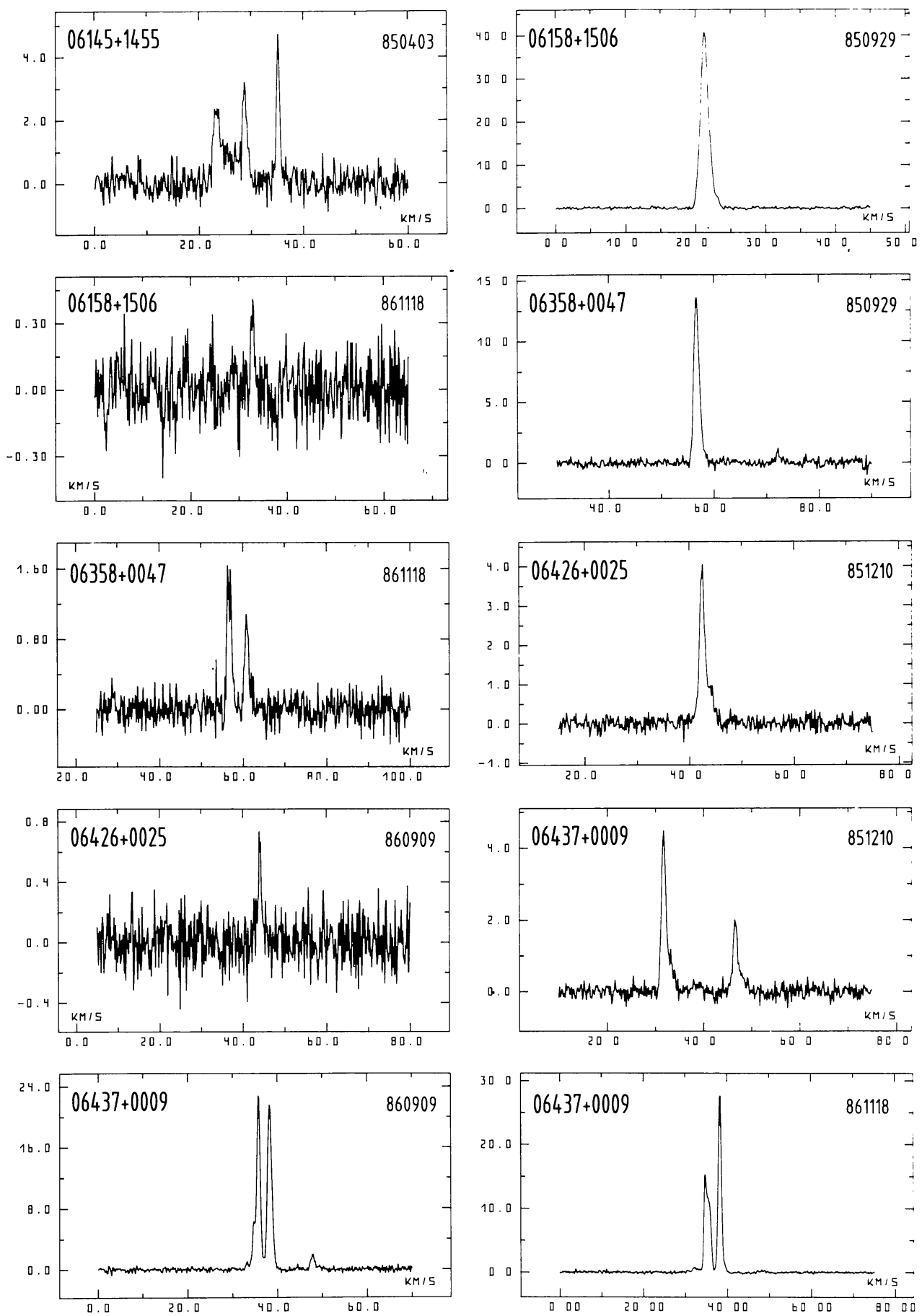

Fig. 2 (continued)

which is very close to the velocity of the molecular cloud $\left(-32 \mathrm{~km} \mathrm{~s}^{-1}\right)$. This and the fact that $\mathrm{H}_{2} \mathrm{O}$ maser sources often are variable suggest that this line was real.

S211: A similar situation as for the maser near BFS 31 occurred for $04324+5106$, which was detected on 851210 and 860212 , but no accurate position could be obtained on 860903 when the maser was found to be weaker than $0.4 \mathrm{Jy}$.
S231: The peak flux density of $05375+3540$ increased substantially between September 1985 and January 1986. This source seems to have only shell components, the velocities of which are not centered exactly at the $\mathrm{CO}$ velocity (the deviation is almost $20 \mathrm{~km} \mathrm{~s}^{-1}$ ).

S259: The CO cloud associated with this $\mathrm{H}$ II region was mapped by Jackson and Sewall (1982). The CO peak is situated about 1'.5 

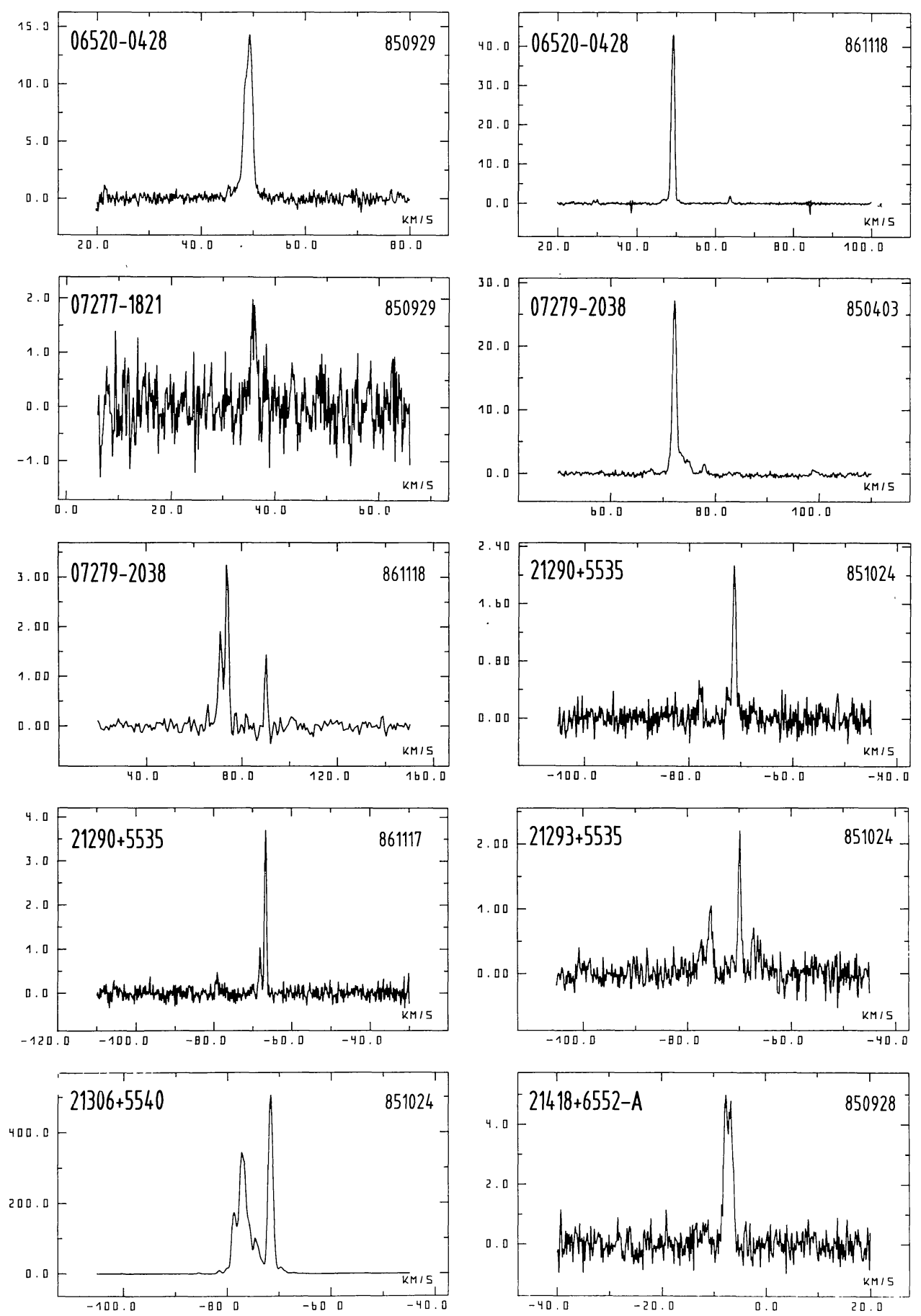

Fig. 2 (continued)

West of the center of the $\mathrm{H}$ II region. The morphology of this area (H II region/IR source/CO cloud) resembles that of the Rosette Nebula (see Blitz and Thaddeus, 1980 and Fig. 5a).

$S 266$ : This is the only region previously studied in the radio continuum with high angular resolution (Israel, 1976). He assumed a distance to this object of $1.5 \mathrm{kpc}$ because no radial velocity was known at that time. His total flux density agrees with the present value, but the map differs from that in Fig. 5b, probably due to the elongated beam used by Israel.

$S$ 286: The VLA map (Fig. 5d) shows several peaks of about the same flux density, one of which is listed in Table 4. The integrated flux density in column 7 is that of the whole source.

$B B W$ 33: This region is split in two parts (A and $\mathrm{B}$; see the catalogue of BBW), separated by approximately $2.4 \mathrm{pc}$. Each 

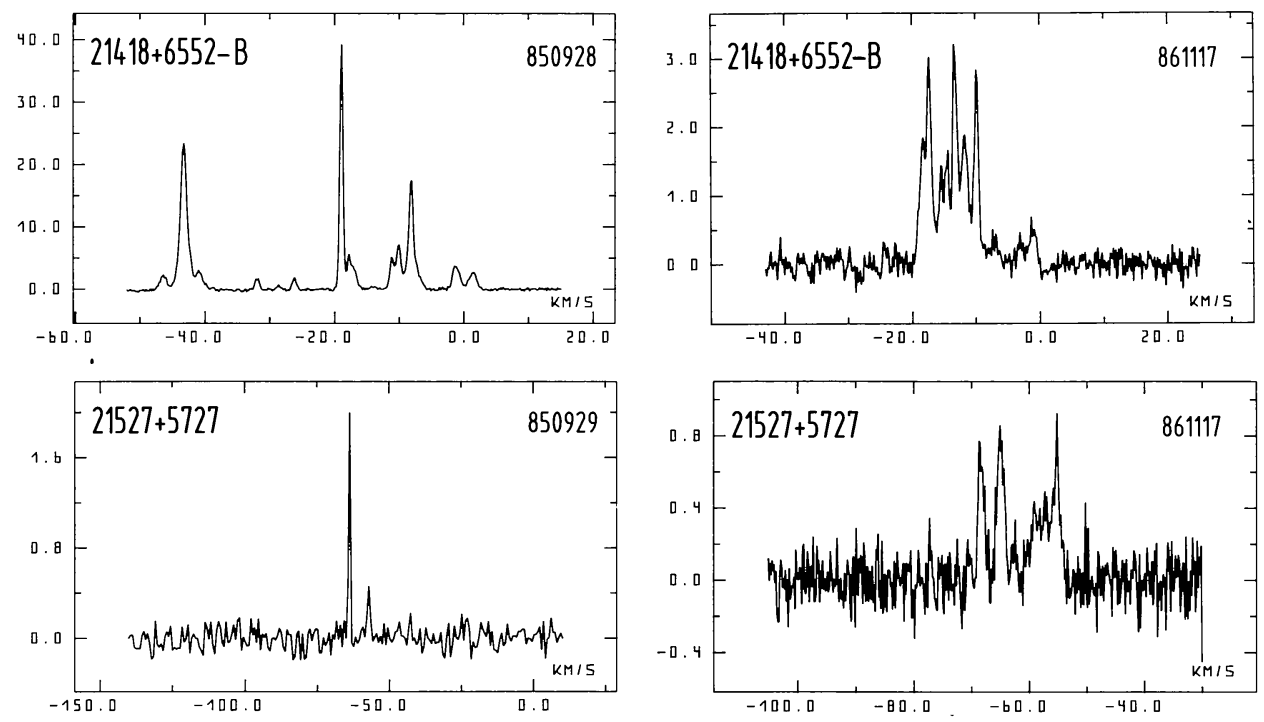

Fig. 2 (continued)
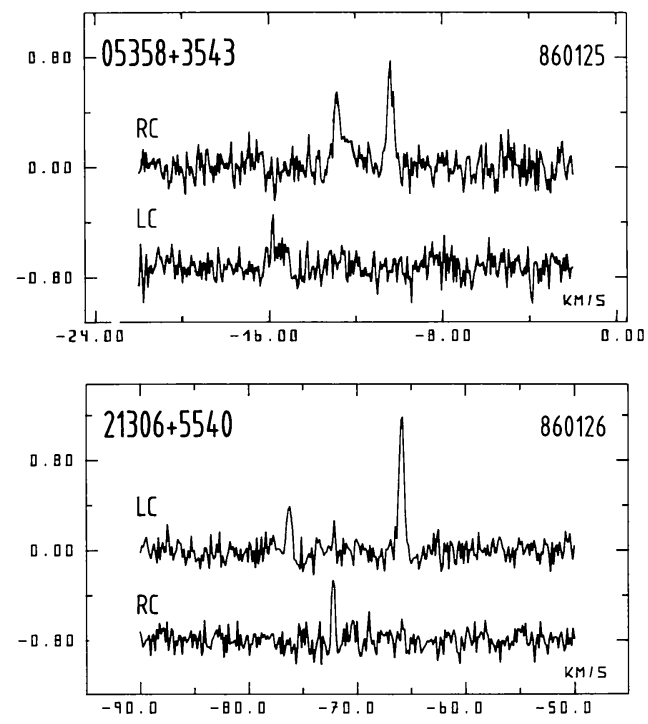

Fig. 3. $1665 \mathrm{MHz} \mathrm{LC}$ and $\mathrm{RC}$ spectra of new $\mathrm{OH}$ masers

consists of a nebulous star. The radio source is centered on $\mathrm{B}$, while the IR source coincides with $\mathrm{A}$; the $\mathrm{H}_{2} \mathrm{O}$ maser is located within $0.5 \mathrm{pc}$ of the latter (see Fig. $5 \mathrm{e}$ ).

$B B W 44 / 45$ : These two objects are very faint nebulosities, with exciting stars either marginally (BBW 44) or not (BBW 45) identifiable. The IR positions coincide with the nebulosities and both exhibit a radio peak. There are blobs of radio emission at the 3-4 $\sigma$ level in between the two radio peaks (Fig. 5f). The total flux density of this emission is $51.5 \mathrm{mJy}$. CO emission at about $75 \mathrm{~km} \mathrm{~s}^{-1}$ is associated with this complex (Brand et al., 1987); the CO emission at BBW 44 exhibits an asymmetry, in the sense of an enhanced red shoulder in the line profiles.

\section{Discussion}

The fact that we detect 15 water masers associated with far infrared sources in the outer Galaxy proves that star formation is going on in this region. That this should be so is not obvious because the physical conditions in the outer Galaxy (the galactic plane with $R>14 \mathrm{kpc}$ ) differ from those in the inner parts or even from those in the solar circle region, seemingly making the conditions for star formation more unfavourable. In this section we first discuss these conditions in more detail and then compare the star forming activity beyond $R=14 \mathrm{kpc}$ with that in other parts of the Galaxy.

\subsection{Physical conditions in the outer Galaxy}

The processes of the formation of $\mathrm{H}$ I cloud complexes, of $\mathrm{H}_{2}$ formation and the triggering of star formation are influenced by various parameters, such as e.g. the relative velocity and frequency of passing density waves, and the numerous and often poorly known physical parameters of the cloud and intercloud medium. It is clear that differences in at least some of these parameters exist between different parts of the Galaxy, but their potential effects on the formation of $\mathrm{HI} / \mathrm{H}_{2}$ clouds and star forming processes are uncertain. Furthermore it is still unknown if the formation of $\mathrm{HI} / \mathrm{H}_{2}$ clouds and stars occurs simultaneously or not. More specifically, it should be investigated, whether there is a larger fraction of molecular clouds without any star formation in the outer than in the inner Galaxy.

Mead (1985) finds that the kinetic temperature of GMC's is generally lower at large $R$ than in other parts of the Galaxy (8 versus $10 \mathrm{~K}$ ), possibly caused by a lower cosmic ray flux (Bloemen et al., 1984). If real, this will affect star formation. However, a lower metallicity could decrease the cooling rate which would have an opposite effect. The metallicity at $R>15 \mathrm{kpc}$ is about a factor 1.5 lower than at the solar circle (Shaver et al., 1983). A lower metallicity could also increase the upper mass limit of the stars formed (Kahn, 1974) and can cause an underestimate of the masses of molecular clouds because the $\mathrm{CO}$ abundance might be smaller than usually assumed. 


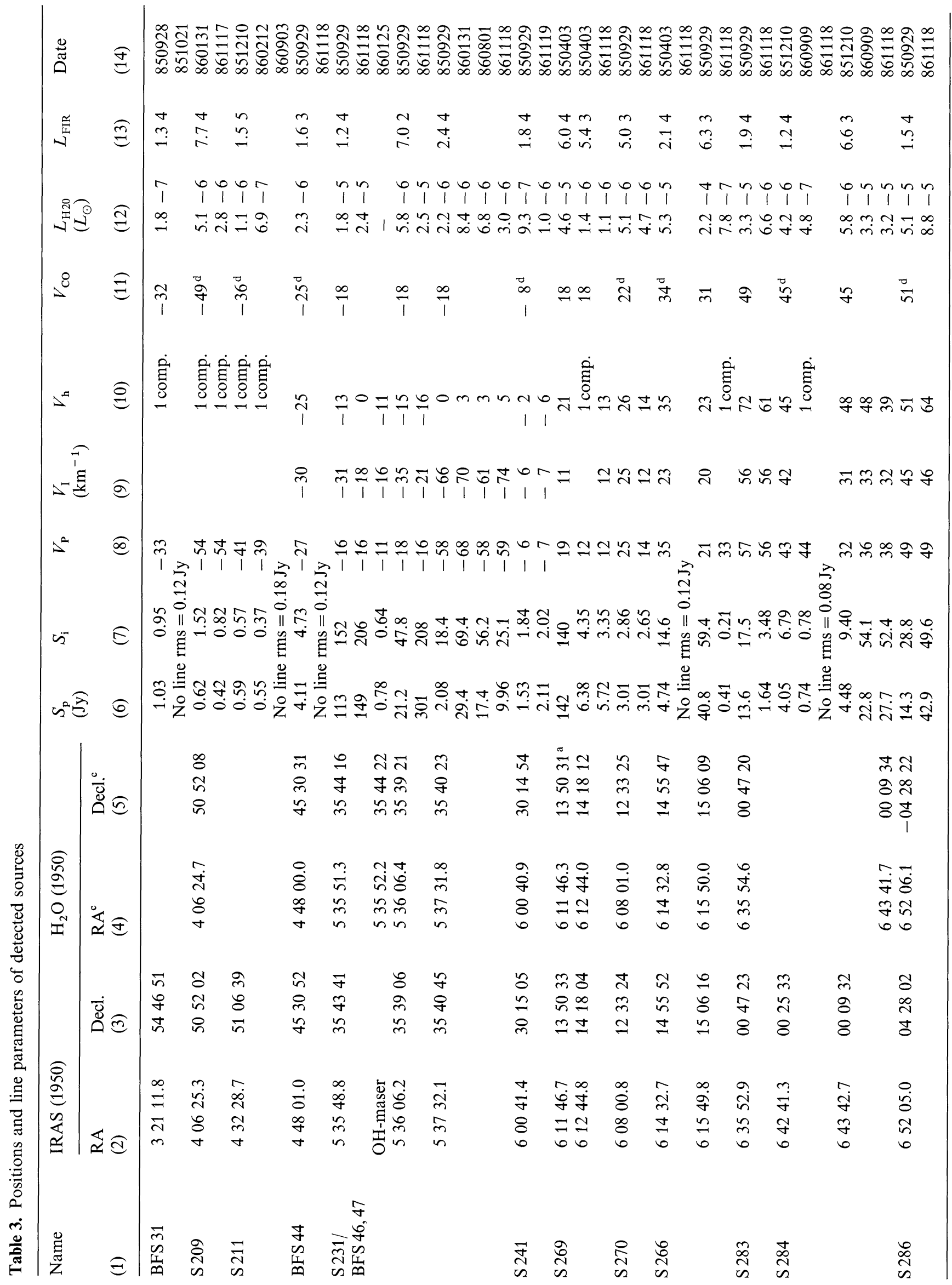




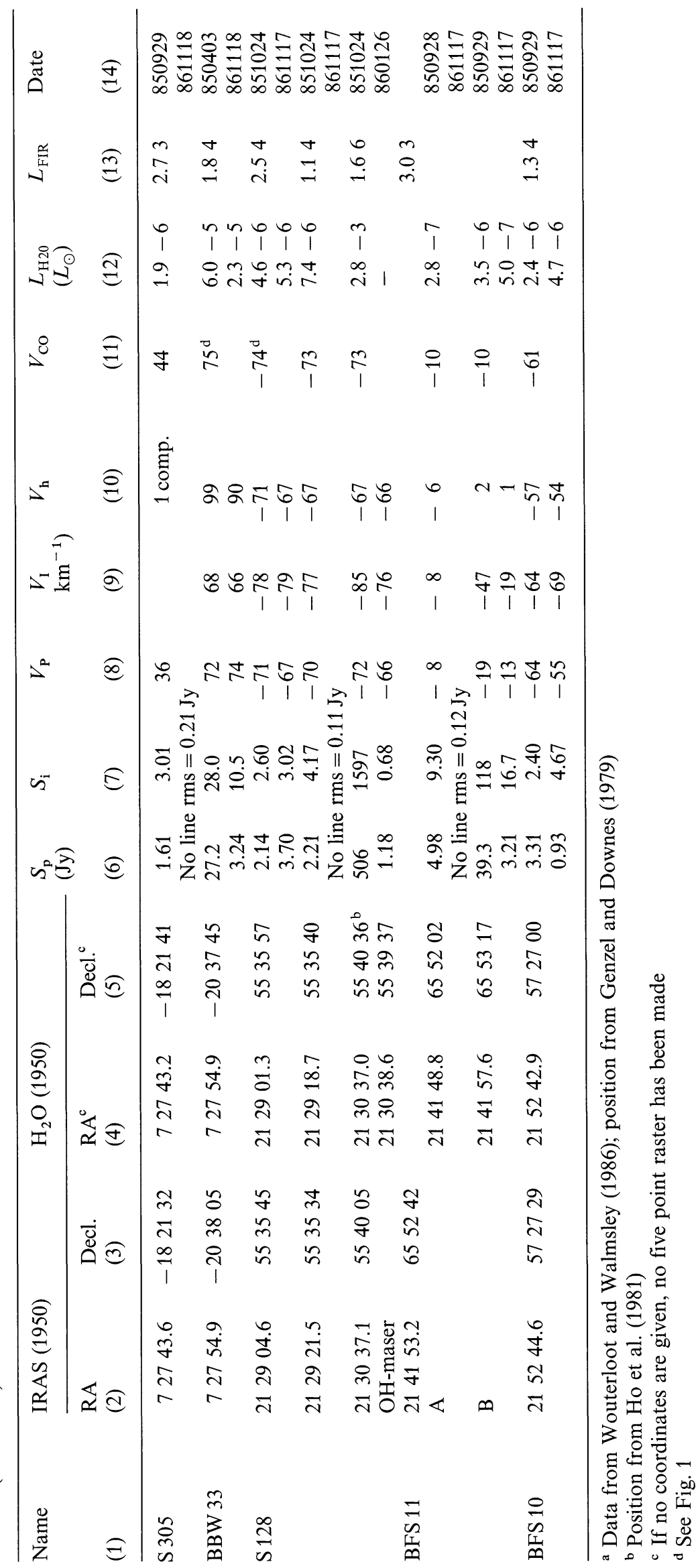



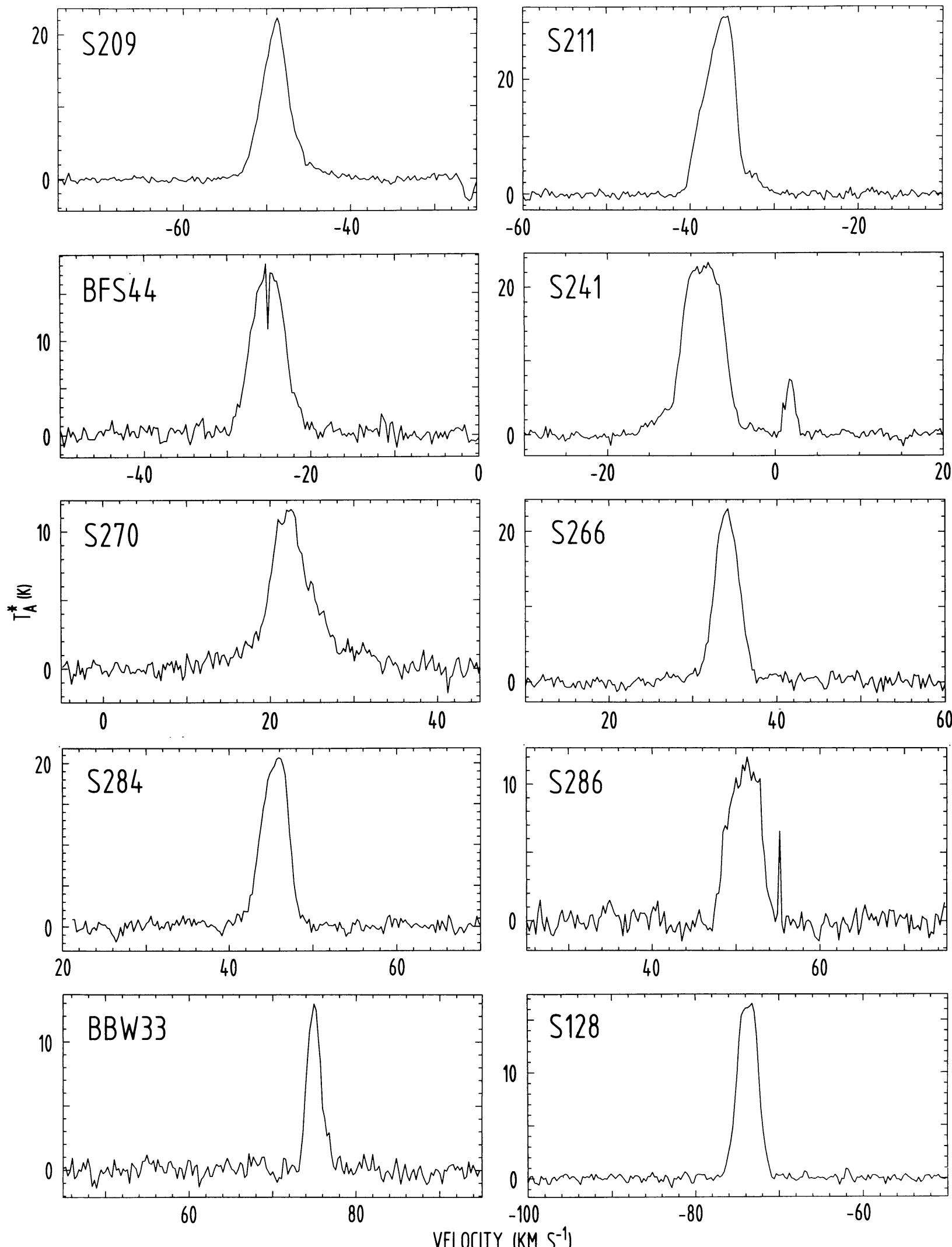

Fig. 4. $115 \mathrm{GHz} \mathrm{CO}$ profiles toward 10 of the $\mathrm{H}_{2} \mathrm{O}$ masers in the outer Galaxy (indicated in column 11 in Table 3). The spectra of BFS 44 and S 286 contain a bad channel. Note the extended linewings in the spectra from S 209 and S 270, two sources at a galactocentric radius of approximately $20 \mathrm{kpc}$ 
Table 4. Radio continuum sources detected with the VLA

\begin{tabular}{|c|c|c|c|c|c|c|c|}
\hline \multirow[b]{2}{*}{ (1) } & \multicolumn{2}{|l|}{ IRAS(1950) } & \multicolumn{2}{|c|}{ Radio (1950) } & \multirow{2}{*}{$\begin{array}{l}\begin{array}{l}S_{\mathrm{P}} \\
(\mathrm{mJy})\end{array} \\
(6)\end{array}$} & \multirow{2}{*}{$\begin{array}{l}\begin{array}{l}S_{\mathrm{I}} \\
(\mathrm{mJy})\end{array} \\
(7)\end{array}$} & \multirow{2}{*}{$\begin{array}{l}\text { Size } \\
\left({ }^{\prime \prime}\right) \\
(8)\end{array}$} \\
\hline & $\begin{array}{l}\text { RA } \\
\text { (2) }\end{array}$ & $\begin{array}{l}\text { Decl. } \\
\text { (3) }\end{array}$ & $\begin{array}{l}\text { RA } \\
\text { (4) }\end{array}$ & $\begin{array}{l}\text { Decl. } \\
\text { (5) }\end{array}$ & & & \\
\hline \multicolumn{8}{|c|}{ S 270} \\
\hline 1 & 060723.5 & 124924 & 060723.6 & 124928 & $22.8 \pm 0.2$ & $31.9 \pm 0.6$ & $\mathrm{nr}$ \\
\hline 2 & no FIR & & 060741.2 & 124611 & $12.3 \pm 0.4$ & $15.1 \pm 0.8$ & $\mathrm{nr}$ \\
\hline 3 & no FIR & & 060756.9 & 123012 & $5.4 \pm 0.1$ & $5.3 \pm 0.1$ & $\mathrm{nr}$ \\
\hline 4 & $\begin{array}{lll}06 & 08 & 0.8\end{array}$ & 123324 & & & $<0.2$ & & \\
\hline \multicolumn{8}{|c|}{ S 259} \\
\hline 1 & 060829.8 & 172710 & 060831.9 & 172710 & $7.6 \pm 0.5$ & $114.7 \pm 9.2$ & 114 \\
\hline 2 & no FIR & & 060849.6 & 172649 & $35.0 \pm 0.4$ & $38.5 \pm 1.2$ & $\mathrm{nr}$ \\
\hline \multicolumn{8}{|c|}{ S 266} \\
\hline 1 & 061432.7 & 145552 & & & $<0.3$ & & \\
\hline 2 & no FIR & & 061433.0 & 145153 & $21.4 \pm 0.2$ & $35.1 \pm 0.6$ & $\mathrm{nr}$ \\
\hline 3 & 061549.8 & 150616 & 061549.8 & 150642 & $9.4 \pm 0.1$ & $10.3 \pm 0.2$ & $\mathrm{nr}$ \\
\hline 4 & no FIR & & 061543.9 & 151328 & $116.4 \pm 0.9$ & $142.1 \pm 2.6$ & $\mathrm{nr}$ \\
\hline 5 & 061552.9 & 151759 & 061553.1 & 151750 & $11.2 \pm 0.7$ & $92.1 \pm 7.1$ & $93 \times 71$ \\
\hline \multicolumn{8}{|c|}{ S 283} \\
\hline 1 & 063552.9 & 004723 & 063552.7 & 004722 & $3.4 \pm 0.1$ & $23.9 \pm 0.2$ & 57 \\
\hline 2 & no FIR & & 063556.5 & 004431 & $18.0 \pm 0.2$ & $32.8 \pm 0.6$ & $\mathrm{nr}$ \\
\hline \multicolumn{8}{|c|}{ S 286} \\
\hline 1 & 065155.0 & -042453 & & & $<0.7$ & & \\
\hline 2 & 065205.0 & -042802 & 065203.7 & -042810 & $1.6 \pm 0.2$ & $58.1 \pm 7.9$ & $200 \times 100$ \\
\hline 3 & no FIR & & 065222.1 & -043610 & $66.2 \pm 4.0$ & $66.2 \pm 3.6$ & $\mathrm{nr}$ \\
\hline \multicolumn{8}{|c|}{ BBW 33} \\
\hline 1 & no FIR & & 072745.0 & -204200 & $4.9 \pm 0.3$ & $4.9 \pm 0.3$ & $\mathrm{nr}$ \\
\hline 2 & 072754.9 & -203805 & 072755.7 & -203835 & $2.6 \pm 0.1$ & $9.3 \pm 0.2$ & $\mathrm{nr}$ \\
\hline 3 & no FIR & & 072758.9 & -204215 & $4.6 \pm 0.2$ & $4.6 \pm 0.2$ & $\mathrm{nr}$ \\
\hline \multicolumn{8}{|c|}{ BBW 44/45 } \\
\hline 1 & 073103.6 & -220105 & 073104.0 & -220050 & $2.0 \pm 0.3$ & $11.3 \pm 2.1$ & $51 \times 31$ \\
\hline 2 & 073111.5 & -220425 & 073111.2 & -220425 & $23.9 \pm 0.2$ & $26.9 \pm 0.4$ & $\mathrm{nr}$ \\
\hline \multicolumn{8}{|c|}{ BBW 66} \\
\hline 1 & 074256.3 & -252351 & & & $<0.9$ & & \\
\hline 2 & no FIR & & 074305.5 & -252907 & $38.8 \pm 0.7$ & $44.0 \pm 1.9$ & $\mathrm{nr}$ \\
\hline 3 & 074318.3 & -253029 & & & $<14.7$ & & \\
\hline
\end{tabular}

Observational evidence for the presence or absence of optical spiral arms beyond the Perseus arm $(R=12-15 \mathrm{kpc})$ is weak because of the small number of objects involved (H II regions, OB associations) and the uncertainty in their distances. H I data indicate possible features with a kinematic $R$ of up to about $20 \mathrm{kpc}$ (Kulkarni et al., 1982; Blitz et al., 1983). Similar features are seen in molecular clouds (Murphy, 1985). Beyond this radius there is no evidence for spiral arms. This weakness or absence of spiral arms is not unexpected (Burton, 1976). Adopting a recent rotation curve (e.g. Brand, 1986; Fich et al., 1987) and a pattern speed of $13.5 \mathrm{~km} \mathrm{~s}^{-1} \mathrm{kpc}^{-1}$, corotation is at about $20 \mathrm{kpc}$. Beyond this radius no $\mathrm{H}$ in regions and no spiral arms are found. Elmegreen (1985) suggests that star formation in an arm is not triggered more than in other areas, but there is only more activity because there is more gas in an arm. This picture would be in agreement with our results which are discussed in the next subsection.

\subsection{The star-forming activity in the outer Galaxy}

The efficiency of star formation is indicated by the fraction of gas mass that is converted into stars. The mass in stars is difficult to estimate and we shall use $\mathrm{H}$ II regions as a first indicator rather than $\mathrm{H}_{2} \mathrm{O}$ masers because the numbers involved are larger and statistical estimates therefore more accurate. For the gas we shall first take only the $\mathrm{H}_{2}$ and later discuss the fractions of $\mathrm{H} \mathrm{I}$ and $\mathrm{H}_{2}$. In order to compare the star forming process in the outer Galaxy with that near the Sun we computed the ratio of the number of $\mathrm{H}$ II regions per $\mathrm{kpc}^{2}$ and the $\mathrm{H}_{2}$ surface density $\left(N(\mathrm{H} \mathrm{II}) / \sigma\left(\mathrm{H}_{2}\right)\right)$. The surface density of $\mathrm{H}$ II regions was obtained from BFS and it was assumed that all objects seen near the Sun could also be found on POSS or SRC-J plates in searches such as made by BFS and BBW if they were located at $R=15 \mathrm{kpc}$. From this we obtain $21 \mathrm{H} \mathrm{II}$ regions per $\mathrm{kpc}^{2}$ near the Sun and $1.4 \mathrm{H}$ II regions per $\mathrm{kpc}^{2}$ at 


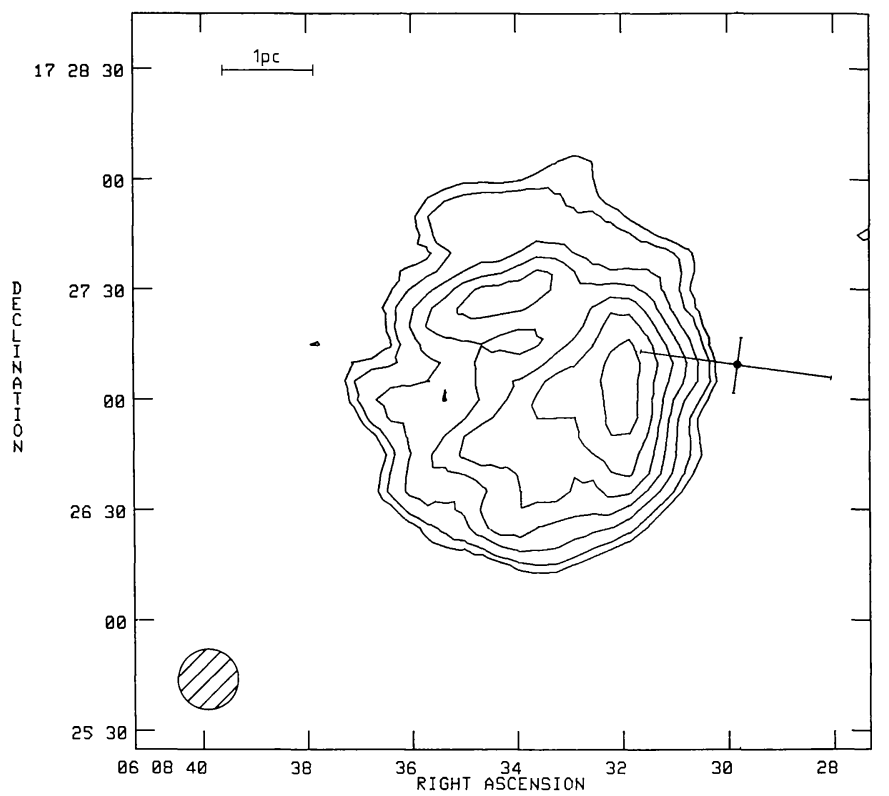

Fig. 5a. Map of $6 \mathrm{~cm}$ radio continuum emission of S 259. Contour values are $1.5,2,2.5,3.5,4,4.5 \mathrm{mJy}$ per beam. The filled circle indicates the position of an IRAS source

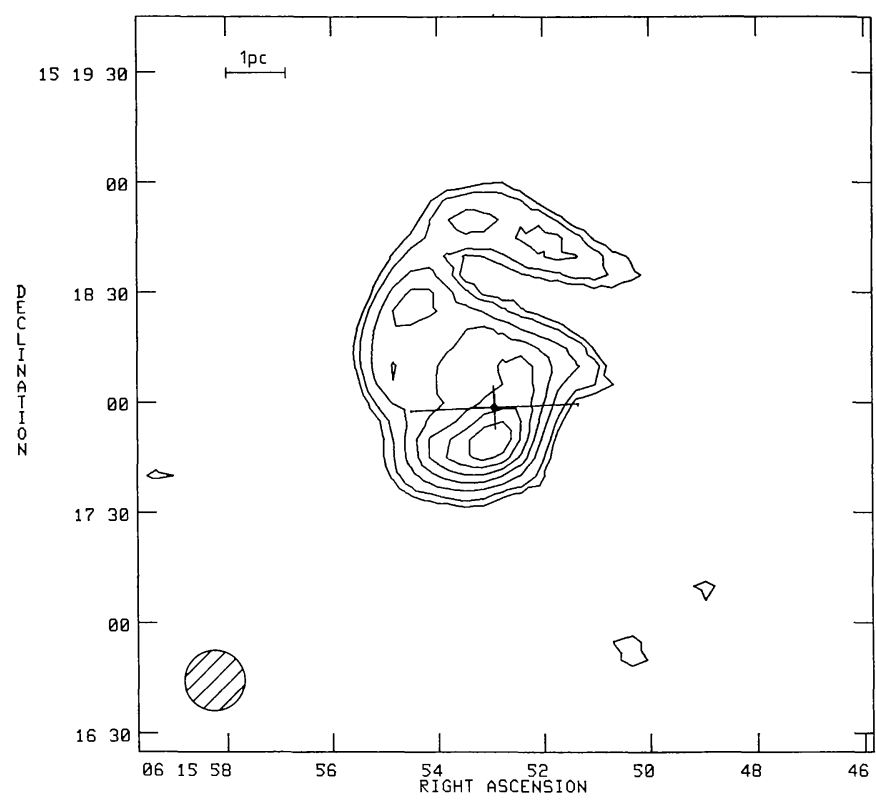

Fig. 5b. As Fig. 5a for S 266. Contour values are 2.1, 2.8, 3.5, 4.2, 4.9, 5.6, $6.3 \mathrm{mJy}$ per beam

$R=15 \mathrm{kpc}$. To estimate the $\mathrm{H}_{2}$ surface density we used data by Sanders et al. (1984), Dame and Thaddeus (1985), and Maddalena (1986). At the solar circle, Dame and Thaddeus find $2 M_{\odot} / \mathrm{pc}^{2}$. Sanders et al. obtain 5.2 and $0.23 M_{\odot} / \mathrm{pc}^{2}$ at $R=10 \mathrm{kpc}$ and $R=14-15 \mathrm{kpc}$ respectively. Scaling these numbers with the ratio $N_{\mathrm{H}_{2}} / W_{\text {CO }}$ of $2.610^{20}$, used by Dame and Thaddeus, they become 3.8 and $0.17 M_{\odot} / \mathrm{pc}^{2}$ respectively. Maddalena finds

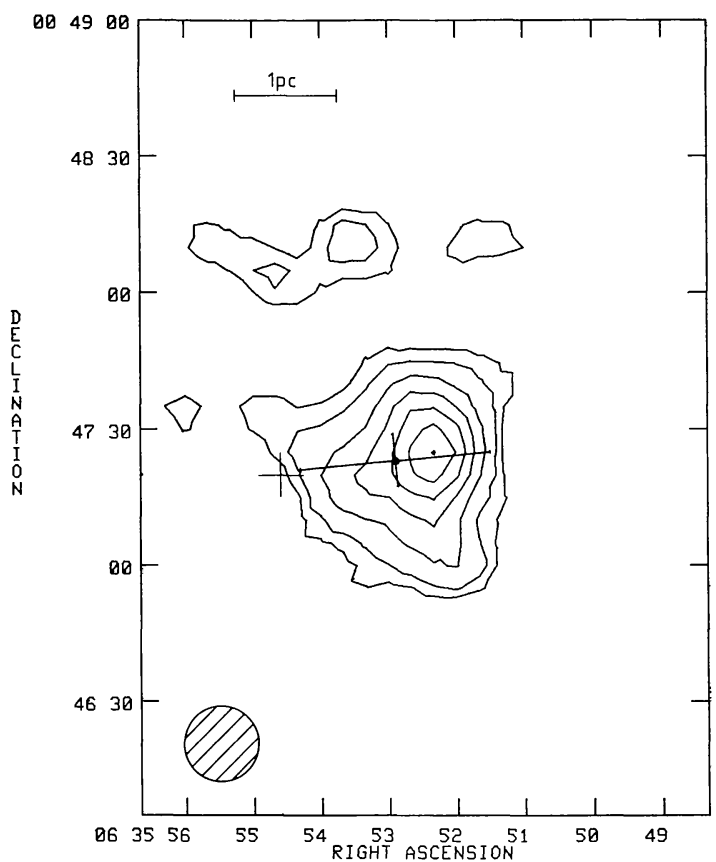

Fig. 5c. As Fig. 5 a for $\mathrm{S} 283$. The cross indicates the position of the $\mathrm{H}_{2} \mathrm{O}$ maser. Contour values are $0.9,1.2,1.8,2.4,3.0,3.6,4.2 \mathrm{mJy}$ per beam

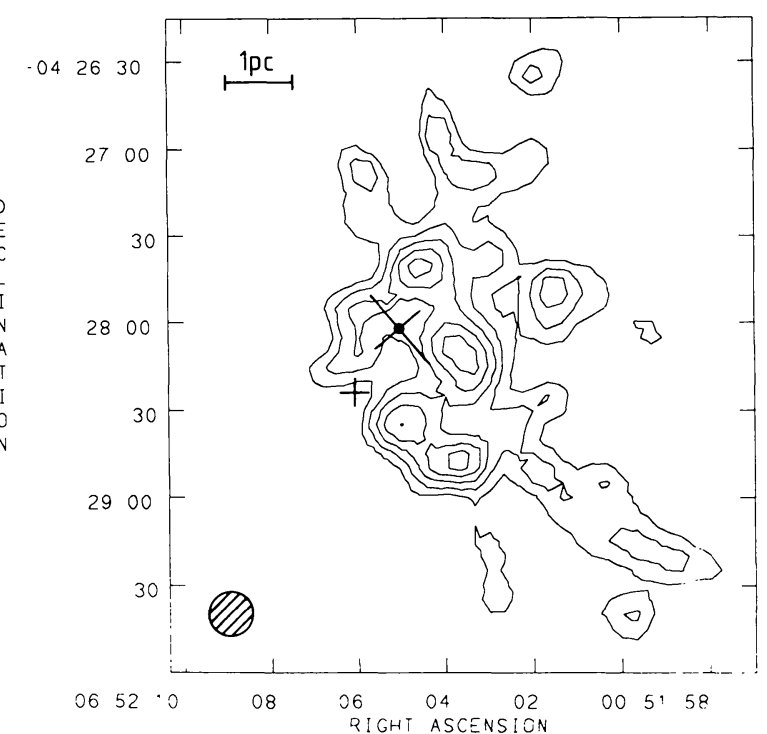

Fig. 5d. As Fig. 5a for S 286. Contour values are 0.6, 0.8, 1.0, 1.2,1.4 mJy per beam

$0.06 M_{\odot} / \mathrm{pc}^{2}$ for $R=13-15 \mathrm{kpc}$, but suggests that it might be a factor of two too low because a part of the material has been missed in his survey. When this correction is applied and we use the mean values of the various authors, we obtain $\mathrm{H}_{2}$ surface densities of $2.9 M_{\odot} / \mathrm{pc}^{2}$ near the Sun and $0.145 M_{\odot} / \mathrm{pc}^{2}$ at $R=15 \mathrm{kpc}$. The the ratio $N(\mathrm{H} \mathrm{II}) / \sigma\left(\mathrm{H}_{2}\right)$ is $7.2(\mathrm{pc} / \mathrm{kpc})^{2} / M_{\odot}$ near the Sun and $9.9(\mathrm{pc} / \mathrm{kpc})^{2} / M_{\odot}$ at $R=15 \mathrm{kpc}$. These ratios are 


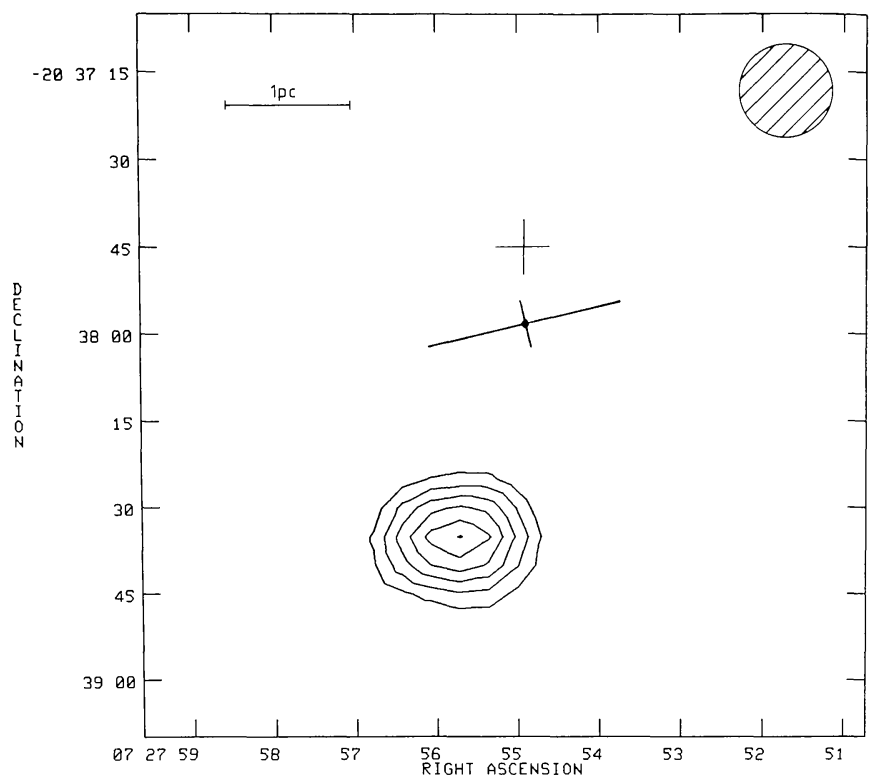

Fig. 5e. As Fig. 5a for BBW 33. Contour values are 1.2, 1.6, 2.0, 2.4, 2.8, $3.2 \mathrm{mJy}$ per beam

equal within the errors. If the star formation process would be very inefficient at large $R$, one would expect fewer $\mathrm{H}$ II regions per unit mass of $\mathrm{H}_{2}$ than near the Sun. This however, is not the case.

We now compare star formation processes in the Perseus arm and in the outer Galaxy. For the Perseus arm we took the IR data from Wouterloot and Walmsley (1986) (who studied an area near $l=110^{\circ}$ ) and the $\mathrm{H}$ II data from BFS, considering the regions mentioned by BFS as constituting a "kinematically distinct complex" as one region. The $\mathrm{H}_{2} \mathrm{O}$ detection rate of outer Galaxy group 1 sources (omitting observed and detected sources with an upper limit at 60 or $100 \mu \mathrm{m}$; see Wouterloot and Walmsley, 1986) with an IR luminosity larger than $10^{4} L_{\odot}$ and $R>14 \mathrm{kpc}$ is $35 \%(8$ out of 23). This is equal (within the statistical error of $10 \%$ ) to that of sources in the Perseus arm, where a value of $36 \%$ (5 out of 14) is obtained by Wouterloot and Walmsley (1986). The number of FIR sources with IR luminosities larger than $10^{4} L_{\odot}$ per $\mathrm{H}$ II region is $23 / 29=0.8 \pm 0.2$ for the outer Galaxy and $14 / 14=1.0 \pm 0.2$ for the Perseus arm. These results suggest that all molecular clouds once formed show the same star formation activity and that there is no difference between clouds in the region near the Sun and in the outer Galaxy.

The results obtained above and the apparently similar mass spectrum of molecular clouds in both parts of the Galaxy (Terebey et al., 1986) are surprising in view of the large variation in the ratio of $\mathrm{HI}$ and $\mathrm{H}_{2}$ surface densities. Between $R=10$ and $R=20 \mathrm{kpc}$ the $\mathrm{H}$ i surface density stays constant at a level of $2.9 M_{\odot} / \mathrm{pc}^{2}$ (Blitz et al., 1983), but the ratio of $\mathrm{H}_{2}$ to $\mathrm{H}$ I surface density changes from about unity near the Sun to 0.05 at $R=15 \mathrm{kpc}$. If for the scale height of the molecular gas we adopt that of the $\mathrm{H}$ II regions, it changes from $100 \mathrm{pc}$ at $R=10 \mathrm{kpc}$ to an average value of $300 \mathrm{pc}$ at $R>14.5 \mathrm{kpc}$ (Fich and Blitz, 1984). For the $\mathrm{H} \mathrm{I}$, the change in scale height is almost the same (Kulkarni et al., 1982). It is $250 \mathrm{pc}$ at $R=10 \mathrm{kpc}$ and $750 \mathrm{pc}$ at $R=20 \mathrm{kpc}$; the average value at $R>14.5 \mathrm{kpc}$ is about $600 \mathrm{pc}$. This means that the decrease in volume density for $\mathrm{H}_{2}$ is even larger than the decrease in surface density and also the volume density of $\mathrm{H}_{\mathrm{I}}$ is decreasing. Possibly

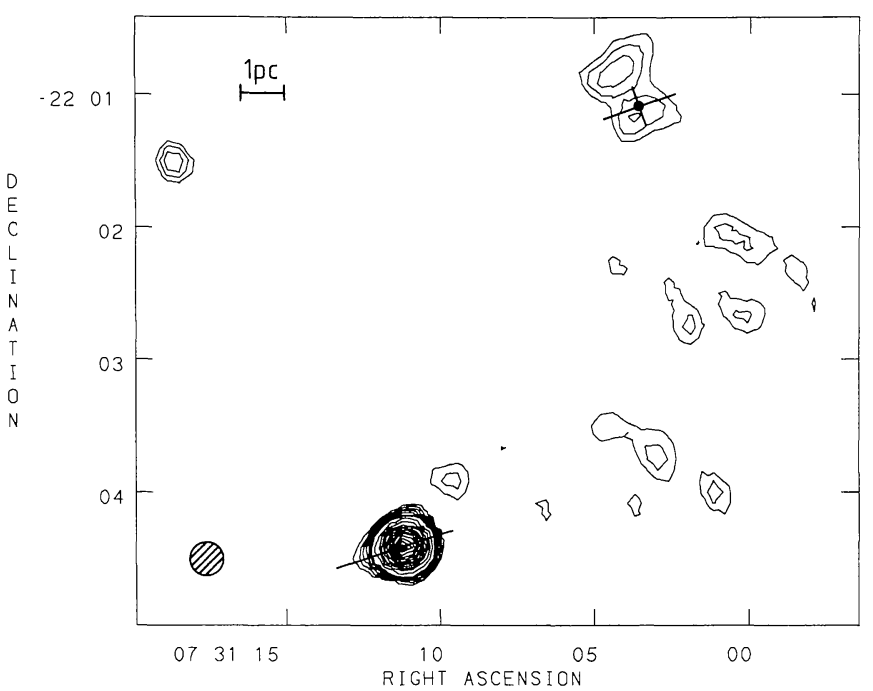

Fig. 5f. As Fig. 5a for BBW 44, 45. Contour values are $0.9-3.0 \mathrm{mJy}$ in steps of 0.3 and $4.5-22.5 \mathrm{mJy}$ per beam in steps of 1.5

the differences in star formation activity between the areas are only due to the number of $\mathrm{H}_{2}$ clouds formed.

There appears to exist another observationally pronounced difference between the outer Galaxy and the solar circle area: the size of the $\mathrm{H}$ in regions. Fich and Blitz (1984) find that the diameter of $\mathrm{H}$ II regions is significantly smaller at $R>14 \mathrm{kpc}$ than at $R<14 \mathrm{kpc}$. The difference seems to be at least a factor of two. There are several possible explanations for this effect: selection effects (this is unlikely since at large $R$ many large $\mathrm{H}$ in regions have probably not been missed) or differences in the properties of the molecular clouds or the exciting stars due to a different IMF. Optical information is incomplete for the outer Galaxy: exciting stars are not identified in most cases (with the exception of some regions discussed by Brand (1986) and Moffat et al. (1979)). Using these data together with the results of Georgelin (1975) in the inner Galaxy it is seen that in both parts of the Galaxy small $(<20 \mathrm{pc})$ $\mathrm{H}$ II regions generally are excited by $\mathrm{B}$ and late $\mathrm{O}$ stars, whereas large regions contain one of more earlier $\mathrm{O}$ stars. Garmany et al. (1982) found that the IMF is steeper for $R>12 \mathrm{kpc}$ than in the solar neighbourhood. Such an effect should also be observable in the distribution of the luminosity of the infrared sources associated with the H II regions. Table 6 shows this for group 1 sources with $R>14 \mathrm{kpc}$ and $L>10^{4} L_{\odot}$ in comparison with the Perseus arm sources (see Wouterloot and Walmsley, 1986). The upper end of the distribution of stellar luminosities (and hence masses; $M>6.5 M_{\odot}$ for a single ZAMS star) that are formed in the two areas is the same within the errors. However the differences found by Garmany et al. occur only for stars with $M>30 M_{\odot}$ (i.e. $L>10^{5} L_{\odot}$ ), where our numbers are too small to be conclusive.

Because the $\mathrm{H}_{2}$ surface density is at least a factor 20 lower at $R>15 \mathrm{kpc}$ than in the solar neighbourhood while the mass spectrum is nearly the same, it is possible that in the outer Galaxy the number of clouds with masses in the upper end of the local range of cloud masses is very small. Smaller and therefore less massive clouds form fewer stars and therefore in most cases no 
Table 5. Derived parameter of continuum sources

\begin{tabular}{|c|c|c|c|c|c|c|c|}
\hline (1) & $\begin{array}{l}u \\
\left(\mathrm{pc} / \mathrm{cm}^{2}\right) \\
(2)\end{array}$ & $\begin{array}{l}\text { Size } \\
(\mathrm{pc}) \\
(3)\end{array}$ & $\begin{array}{l}n_{\mathrm{e}} \\
\left(\mathrm{cm}^{-3}\right) \\
(4)\end{array}$ & $\begin{array}{l}L_{\mathrm{FIR}} \\
\left(L_{\odot}\right) \\
(5)\end{array}$ & $\begin{array}{l}\text { Sp type } \\
(6)\end{array}$ & $\begin{array}{l}d \\
(\mathrm{kpc}) \\
(7)\end{array}$ & (8) \\
\hline \multicolumn{8}{|c|}{ S 270} \\
\hline 1 & $18.3 \pm 3.1$ & $<0.7$ & $>300$ & $1.210^{5}$ & B0 III & \multirow[t]{4}{*}{$8.8 \pm 2.2$} & \multirow[t]{4}{*}{$\mathrm{k}$} \\
\hline 2 & $14.2 \pm 2.4$ & $<0.7$ & $>206$ & - & $\mathrm{B} 0-0.5 \mathrm{~V}$ & & \\
\hline 3 & $1.2 \pm 1.7$ & $<0.7$ & $>122$ & - & B $0.5 \mathrm{~V}$ & & \\
\hline 4 & - & - & - & $5.010^{3}$ & $\mathrm{~B} 2 \mathrm{~V}$ & & \\
\hline \multicolumn{8}{|c|}{ S 259} \\
\hline 1 & $26.9 \pm 4.6$ & 4.6 & 219 & $3.410^{4}$ & $09.5 \mathrm{~V}_{0}$ & \multirow{2}{*}{$8.3 \pm 2.1$} & \multirow[t]{2}{*}{$\mathrm{p}$} \\
\hline 2 & $18.7 \pm 3.1$ & $<0.6$ & $>339$ & - & $\mathrm{B} 0 \mathrm{~V}$ & & \\
\hline \multicolumn{8}{|c|}{ S 266} \\
\hline 1 & - & - & - & $2.110^{4}$ & $\mathrm{~B} 0.5 \mathrm{~V}$ & \multirow[t]{5}{*}{$12.6 \pm 3.1$} & \multirow[t]{5}{*}{$\mathrm{k}$} \\
\hline 2 & $23.9 \pm 4.0$ & $<1.0$ & $>263$ & - & $\mathrm{B} 0 \mathrm{~V}$ & & \\
\hline 3 & $15.9 \pm 2.7$ & $<1.0$ & $>142$ & $6.310^{3}$ & $\mathrm{~B} 0.5 \mathrm{~V}_{0}$ & & \\
\hline 4 & $38.2 \pm 6.4$ & $<1.0$ & $>528$ & - & $\mathrm{O} 8 \mathrm{~V}$ & & \\
\hline 5 & $33.0 \pm 5.6$ & $5.7 \times 4.3$ & 190 & $1.110^{5}$ & B0 III & & \\
\hline \multicolumn{8}{|c|}{ S 283} \\
\hline 1 & $17.0 \pm 1.6$ & 2.5 & 135 & $1.910^{4}$ & $\mathrm{~B} 0 \mathrm{~V}_{0}$ & \multirow{2}{*}{$9.1 \pm 1.3$} & \multirow[t]{2}{*}{$\mathrm{p}$} \\
\hline 2 & $18.8 \pm 1.7$ & $<0.7$ & $>299$ & - & $\mathrm{B} 0 \mathrm{~V}$ & & \\
\hline \multicolumn{8}{|c|}{ S 286} \\
\hline 1 & - & - & - & $4.510^{3}$ & $\mathrm{~B} 2 \mathrm{~V}$ & \multirow[t]{3}{*}{$8.8 \pm 2.2$} & \multirow[t]{3}{*}{$\mathrm{k}$} \\
\hline 2 & $22.3 \pm 3.9$ & $8.5 \times 4.3$ & 132 & $1.510^{4}$ & $\mathrm{~B} 0 \mathrm{~V}_{0}$ & & \\
\hline 3 & $33.2 \pm 5.6$ & $<0.6$ & $>432$ & - & $09-9.5 \mathrm{~V}$ & & \\
\hline \multicolumn{8}{|c|}{ BBW 33} \\
\hline 1 & $10.4 \pm 2.9$ & $<0.7$ & $>155$ & - & $\mathrm{B} 0.5 \mathrm{v}$ & \multirow[t]{3}{*}{$9.6 \pm 3.7$} & \multirow[t]{3}{*}{$\mathrm{p}$} \\
\hline 2 & $12.9 \pm 3.3$ & $<0.7$ & $>112$ & $1.810^{4}$ & $\mathrm{~B} 0-0.5 \mathrm{~V}_{0}$ & & \\
\hline 3 & $10.2 \pm 2.6$ & $<0.7$ & $>109$ & - & $\mathrm{B} 0.5 \mathrm{~V}$ & & \\
\hline \multicolumn{8}{|c|}{ BBW 44/45 } \\
\hline 1 & $15.0 \pm 2.7$ & $2.7 \times 1.6$ & 100 & $5.010^{4}$ & $\mathrm{~B} 0 \mathrm{~V}$ & \multirow{2}{*}{$11.0 \pm 2.7$} & \multirow[t]{2}{*}{$\mathrm{k}$} \\
\hline 2 & $20.0 \pm 3.3$ & $<0.9$ & $>246$ & $1.210^{5}$ & B0 III & & \\
\hline \multicolumn{8}{|c|}{ BBW 66} \\
\hline 1 & - & - & - & $6.710^{3}$ & $\mathrm{~B} 1.5 \mathrm{~V}$ & \multirow[t]{3}{*}{$4.1 \pm 0.7$} & \multirow[t]{3}{*}{$\mathrm{p}$} \\
\hline 2 & $11.8 \pm 1.3$ & $<0.3$ & $>517$ & - & B $0-0.5 \mathrm{~V}$ & & \\
\hline 3 & - & - & - & $1.110^{3}$ & $>\mathrm{B} 3 \mathrm{~V}$ & & \\
\hline
\end{tabular}

Table 6. Comparison of the luminosity distribution in the outer Galaxy (OG) and Perseus arm (PA)

\begin{tabular}{lrr}
\hline $\log L$ & $N_{\mathrm{OG}}$ & $N_{\mathrm{PA}}$ \\
\hline 4 & 23 & 16 \\
4.5 & 9 & 7 \\
5 & 5 & 1 \\
5.5 & 0 & 1 \\
\hline
\end{tabular}

massive early $\mathrm{O}$ type stars (even if the IMF is not steeper in the outer Galaxy), which may thus explain the small fraction of large $\mathrm{H}$ II regions in the outer Galaxy.

\section{Conclusions}

We have studied the star forming process in a part of the Galaxy at a large $(>14 \mathrm{kpc})$ distance from the galactic centre by searching for $\mathrm{H}_{2} \mathrm{O}$ maser emission at the position of IRAS sources and by observing eight distant $\mathrm{H}_{\mathrm{II}}$ regions with the VLA. We found $15 \mathrm{H}_{2} \mathrm{O}$ masers in the outer Galaxy. The detection rate of $\mathrm{H}_{2} \mathrm{O}$ emission is the same as in other parts of the Galaxy. Radio emission was found associated with all but one of the optical objects and all but one of the luminous $\left(L>310^{3} L_{\odot}\right)$ IRAS sources. The excitation parameters of the regions studied with the VLA are consistent with excitation by early B-type stars.

The rate of star formation per unit mass of $\mathrm{H}_{2} \mathrm{O}$ at large $\mathrm{R}$ is similar to that in the solar neighbourhood. This means that once $\mathrm{H}_{2}$ clouds are formed they start forming stars independent of their location in the Galaxy. The small number of $\mathrm{H}$ II regions in the outer Galaxy is mainly due to the low $\mathrm{H}_{2}$ surface density. Probable causes for this low $\mathrm{H}_{2}$ surface density are the closeness of corotation, the decreasing volume density and/or the increasing scale-height of $\mathrm{HI}$.

Acknowledgement. JB thanks Marc Oort (Leiden) for introduction to and help with the AIPS reduction programs. 


\section{References}

Baars, J.W. M., Genzel, R., Paulini-Toth, I.I.K., Witzel, A.: 1977, Astron. Astrophys. 61, 99

Blitz, L.: 1980, in Giant Molecular Clouds in the Galaxy, eds. P.M. Solomon, M.G. Edmunds, p. 1

Blitz, L., Fich, M., Stark, A. A.: 1982, Astrophys. J. Suppl. 49, 183

Blitz, L., Fich, M., Kulkarni, S.: 1983, Science 220, 1233

Blitz, L., Thaddeus, P.: 1980, Astrophys. J. 241, 676

Bloemen, J.B.G.M., Bennet, K., Bignami, G.F., Blitz, L., Caraveo, P.A., Gottwald, M., Hermsen, W., Lebrun, F., Mayer-Hasselwander, H.A., Strong, A.W.: 1984, Astron. Astrophys. 135, 12

Brand, J.: 1986, Ph.D. Thesis, Leiden University

Brand, J., Blitz, L., Wouterloot, J.G. A.: 1986, Astron. Astrophys. Suppl. 65, 537

Brand, J., Blitz, L., Wouterloot, J.G.A., Kerr, F.J.: 1987, Astron. Astrophys. Suppl. 68, 1 (Erratum in ibid. 69, 343)

Burton, W. B.: 1976, Ann. Rev. Astron. Astrophys. 14, 275

Caswell, J.L., Batchelor, R.A., Forster, J.R., Wellington, K.J.: 1983, Australian J. Phys. 36, 443

Dame, T.M., Thaddeus, P.: 1985, Astrophys. J. 297, 751

Elmegreen, B.C.: 1985, in Birth and Evolution of Massive Stars and Stellar Groups, eds. W. Boland, H. van Woerden, p 227

Fich, M.: 1983, Ph.D. Thesis, Univ. of California, Berkeley

Fich, M., Blitz, L.: 1984, Astrophys. J. 279, 125

Fich, M., Blitz, L., Stark, A.A.: 1987 (in preparation)

Garmany, C.D., Conti, P.S., Chiosi, C.: 1982, Astrophys. J. 263, 777

Genzel, R., Downes, D.: 1979, Astron. Astrophys. 72, 234
Georgelin, Y.M.: 1975, Ph.D. Thesis, Université de Provence

Henkel, C., Haschick, A.D., Güsten, R.: 1986, Astron. Astrophys. 165, 197

Ho, P.T.P., Haschick, A.D., Israel, F.P.: 1981, Astrophys. J. 243, 526

Israel, F.P.: 1976, Astron. Astrophys. 52, 175

Jackson, P.D., Sewall, J.R.: 1982, in Regions of Recent Star Formation, eds. R.S. Roger, P.E. Dewdney, p 221

Kahn, F.D.: 1974, Astron. Astrophys. 37, 149

Kulkarni, S. R., Blitz, L., Heiles, C.: 1982, Astrophys. J. 259, L 63

Maddalena, R.J.: 1986, Ph.D. Thesis, Columbia University

Maslowski, J., Paulini-Toth, I.I.K., Witzel, A., Kühr, H.: 1984, Astron. Astrophys. 141, 376

Mead, M. N.: 1985, Bull. Am. Astron. Soc. 17, 870

Moffat, A.F.J., FitzGerald, M.P., Jackson, P.D.: 1979, Astron. Astrophys. Suppl. 38, 197

Murphy, D.C.: 1985, Ph.D. Thesis, University of Texas

Panagia, N.: 1973, Astron. J. 78, 929

Sanders, D. B., Solomon, P.M., Scoville, N.Z.: 1984, Astrophys. J. 276, 182

Shaver, P.A., McGee, R.X., Newton, L.M., Danks, A.C., Pottasch, S.R.: 1983, Monthly Notices Roy. Astron. Soc. 204, 53

Tereby, S., Fich, M., Blitz, L., Henkel, C.: 1986, Astrophys. J. 308, 357

Wall, J.V., Scheuer, P.A.G., Paulini-Toth, I.I.K., Witzel, A.: 1982, Monthly Notices Roy. Astron. Soc. 198, 221

Wilson, T.L., Serabyn, E., Henkel, C.: 1986, Astron. Astrophys. 167, L 17

Wouterloot, J.G.A., Walmsley, C.M.: 1986, Astron. Astrophys. 168, 237 